\title{
Risk-Sensitive Control and Dynamic Games for Partially Observed Discrete-Time Nonlinear Systems
}

\author{
Matthew R. James, Member, IEEE, John S. Baras, Fellow, IEEE, and Robert J. Elliott
}

\begin{abstract}
In this paper we solve a finite-horizon partially observed risk-sensitive stochastic optimal control problem for discrete-time nonlinear systems and obtain small noise and small risk limits. The small noise limit is interpreted as a deterministic partially observed dynamic game, and new insights into the optimal solution of such game problems are obtained. Both the risk-sensitive stochastic control problem and the deterministic dynamic game problem are solved using information states, dynamic programming, and associated separated policies. A certainty equivalence principle is also discussed. Our results have implications for the nonlinear robust stabilization problem. The small risk limit is a standard partially observed risk-neutral stochastic optimal control problem.
\end{abstract}

\section{INTRODUCTION}

$\mathbf{R}$ ECENT interest in risk-sensitive stochastic control problems is due in part to connections with $H_{\infty}$ or robust control problems and dynamic games. The solution of a risksensitive problem leads to a conservative optimal policy, corresponding to the controller's aversion to risk.

For linear/quadratic risk-sensitive problems with full state information, Jacobson [17] established the connection with dynamic games. The analogous nonlinear problem was studied recently, and a dynamic game is obtained as a small noise limit [7], [12], [18], [27]. A risk-neutral stochastic control problem obtains as a small risk limit [7], [18].

Whittle [26] solved the discrete-time linear/quadratic risksensitive stochastic control problem with incomplete state information and characterized the solution in terms of a certainty equivalence principle. The analogous continuoustime problem was solved by Bensoussan and van Schuppen [4], where the problem was converted to an equivalent one with full state information. A transformation technique has also been used to solve partially observed linear/quadratic $H_{\infty}$ and dynamic game problems [8], [23], [24], and others). The nonlinear continuous-time partially observed risk-sensitive stochastic control problem was considered by Whittle [28], and an approximate solution was stated using a certainty equivalence principle when the noise is small; these results are not rigorous, but are very insightful.

Manuscript received January 8, 1993; revised May 8, 1993. Recommended by Associate Editor V. Solo. This work was supported in part by Grant NSFD CDR 8803012 through the Engineering Research Centers Program.

M. R. James is with the Department of Systems Engineering and Cooperative Research Centre for Robust and Adaptive Systems, Australian National University, Canberra, ACT 0200, Australia.

J. S. Baras is with the Department of Electrical Engineering and Institute for Systems Research, University of Maryland, College Park, MD 20742.

R. J. Elliott is with the Department of Statistics and Applied Probability, University of Alberta, Edmonton, Alberta T6G 2G1, Canada. IEEE Log Number 9215724.
In this paper we consider the finite-horizon partially observed risk-sensitive stochastic control problem for discretetime nonlinear systems. The solution to this problem together with large deviation limits lead to new insights into the optimal solution of partially observed dynamic game problems and related robust control problems.

The risk-sensitive stochastic control problem is solved by defining an information state and an associated value function and applying dynamic programming (Section II). The dynamic programming equation is a nonlinear infinite-dimensional recursion. Our approach is motivated by the method used by Bensoussan and van Schuppen [4] and the well-known separation method for risk-neutral problems. The resulting optimal controller is expressed in terms of a separated policy through the information state. The derivation of this solution does not involve asymptotic methods.

In Section III we obtain the small noise limit of both the information state and the value function. Logarithmic transformations are employed in each case. The information state limit is similar to large deviations limit results for nonlinear filters [1], [15], [16], [19], [20], where the limit filter can be used as an observer for the limit deterministic system. The limit of the value function also satisfies a nonlinear infinitedimensional recursion, which in Section IV is interpreted as the dynamic programming equation for a deterministic partially observed dynamic game. As a by-product, we obtain an information state and value function for this game and a verification theorem. The optimal output feedback controller for the game is given by a separated policy through the information state. The information state, which depends on the output path, is a function of the state variable and evolves forward in time. The value function is a function of the information state, evolves backwards in time, and determines the optimal control policy. The structure of the controller we obtain is similar to that arising in the solution of the linear/quadratic problem, which involves a pair of Riccati equations, one corresponding to estimation and one to control [14], [26]. We identify a certain saddle point condition under which the certainty equivalence policy proposed by Basar and Bernhard [3] and Whittle [26], [28] is optimal; using our verification theorem. This policy involves the forward dynamic programming recursion for the information state and a backward recursion for the value function for the corresponding dynamic game problem with full state information. This latter value function is (like the information state) a function of the state variable and, consequently, easier to compute. 
The treatment of the robust $H_{\infty}$ nonlinear output feedback control problem using stochastic control formulations leads naturally to the "correct" feedback structure of an "observer" and "controller." Our approach leads directly to this structure through limiting processes which involve large deviation principles. The method clearly establishes the separation of the feedback policy and provides a framework for evaluating practical recipes. This correspondence and application to the discrete-time, nonlinear, robust, output feedback stabilization problem will be described in detail in a different publication [2].

Finally, the small risk limit is evaluated in Section $V$ and shown to be a standard risk-neutral partially observed stochastic control problem. The notion of information state and the use of dynamic programming is well known for riskneutral problems (e.g., [22], [25]). The continuous-time case is discussed in James et al. [21].

\section{THE RISK-SENSITIVE CONTROL PROBLEM}

\section{A. Dynamics}

On a probability space $\left(\Omega, \mathcal{F}, \boldsymbol{P}^{u}\right)$ we consider a risksensitive stochastic control problem for the discrete-time system

$$
\left\{\begin{array}{l}
x_{k+1}^{\epsilon}=b\left(x_{k}^{\epsilon}, u_{k}\right)+w_{k}^{\epsilon}, \\
y_{k+1}^{\epsilon}=h\left(x_{k}^{\epsilon}\right)+v_{k}^{\epsilon}
\end{array}\right.
$$

on the finite time interval $k=0,1,2, \cdots, M$. The process $x^{\epsilon}$ represents the state of the system and is not directly measured. The process $y^{\epsilon}$ is measured and is called the observation process. This observation process can be used to select the control actions $u_{k}$. We will write $x_{k, l}^{\epsilon}$ for the sequence $x_{k}^{\epsilon}, \cdots, x_{l}^{\epsilon}$, etc. $\mathcal{G}_{k}$ and $\mathcal{Y}_{k}$ denote the complete filtrations generated by $\left(x_{0, k}^{\epsilon}, y_{0, k}^{\epsilon}\right)$ and $y_{0, k}^{\epsilon}$ respectively.

We assume:

i) $x_{0}^{\epsilon}$ has density $\rho(x)=(2 \pi)^{-n / 2} \exp \left(-1 / 2|x|^{2}\right)$.

ii) $\left\{w_{k}^{\epsilon}\right\}$ is an $\boldsymbol{R}^{n}$ valued i.i.d noise sequence with density $\psi^{\epsilon}(w)=(2 \pi \epsilon)^{-n / 2} \exp \left(-1 / 2 \epsilon|w|^{2}\right)$.

iii) $y_{0}^{\epsilon}=0$.

iv) $\left\{v_{k}^{\epsilon}\right\}$ is a real-valued i.i.d noise sequence with density $\phi^{\epsilon}(v)=(2 \pi \epsilon)^{-1 / 2} \exp \left(-1 / 2 \epsilon|v|^{2}\right)$, independent of $x_{0}^{\epsilon}$ and $\left\{w_{k}^{\epsilon}\right\}$.

v) $b \in C^{1}\left(\boldsymbol{R}^{n} \times \boldsymbol{R}^{m}, \boldsymbol{R}^{n}\right)$ is bounded and uniformly continuous.

vi) The controls $u_{k}$ take values in $U \subset \boldsymbol{R}^{m}$, assumed compact, and are $\mathcal{Y}_{k}$ measurable. We write $\mathcal{U}_{k, l}$ for the set of such control processes defined on the interval $k, \cdots, l$.

vii) $h \in C\left(\boldsymbol{R}^{n}\right)$ is bounded and uniformly continuous.

The probability measure $\boldsymbol{P}^{u}$ can be defined in terms of an equivalent reference measure $\boldsymbol{P}^{\dagger}$ using the discrete analog of Girsanov's Theorem [11]. Under $\boldsymbol{P}^{\dagger},\left\{y_{k}^{\epsilon}\right\}$ is i.i.d with density $\phi^{\epsilon}$, independent of $\left\{x_{k}^{\epsilon}\right\}$, and $x^{\epsilon}$ satisfies the first equation in (2.1). For $u \in \mathcal{U}_{0, M-1}$

$$
\left.\frac{d \boldsymbol{P}^{u}}{d \boldsymbol{P}^{\dagger}}\right|_{\mathcal{G}_{k}}=Z_{k}^{\epsilon}=\prod_{l=1}^{k} \Psi^{\epsilon}\left(x_{l-1,}^{\epsilon}, y_{l}^{\epsilon}\right)
$$

where

$$
\Psi^{\epsilon}(x, y) \triangleq \exp \left(-\frac{1}{\epsilon}\left[\frac{1}{2}|h(x)|^{2}-h(x) y\right]\right) .
$$

\section{B. Cost}

The cost function is defined for admissible $u \in \mathcal{U}_{0, M-1}$ by

$$
J^{\mu, \epsilon}(u)=\boldsymbol{E}^{u}\left[\exp \frac{\mu}{\epsilon}\left(\sum_{l=0}^{M-1} L\left(x_{l}^{\epsilon}, u_{l}\right)+\Phi\left(x_{M}^{\epsilon}\right)\right)\right]
$$

and the partially observed risk-sensitive stochastic control problem is to find $u^{*} \in \mathcal{U}_{0, M-1}$ such that

$$
J^{\mu, \epsilon}\left(u^{*}\right)=\inf _{u \in \mathcal{U}_{0, M-1}} J^{\mu, \epsilon}(u) .
$$

Here, we assume:

viii) $L \in C\left(\boldsymbol{R}^{n} \times \boldsymbol{R}^{m}\right)$ is nonnegative, bounded, and uniformly continuous.

ix) $\Phi \in C\left(\boldsymbol{R}^{n}\right)$ is nonnegative, bounded, and uniformly continuous.

Remark 2.1: The assumptions i) through ix) are stronger than necessary. For example, boundedness assumption for $b$ can be replaced by a linear growth condition. In addition, a "diffusion" coefficient can be inserted into (2.1). Other choices for the initial density $\rho$ are possible; see Remark 4.1.

The parameters $\mu>0$ and $\epsilon>0$ are measures of risk sensitivity and noise variance. In view of our assumptions, the cost function is finite for all $\mu>0, \epsilon>0$. These parameters will remain fixed throughout Section II.

In terms of the reference measure, the cost can be expressed as

$$
J^{\mu, \epsilon}(u)=\boldsymbol{E}^{\dagger}\left[Z_{M}^{\epsilon} \exp \frac{\mu}{\epsilon}\left(\sum_{l=0}^{M-1} L\left(x_{l}^{\epsilon}, u_{l}\right)+\Phi\left(x_{M}^{\epsilon}\right)\right)\right] .
$$

\section{Information State}

We consider the space $L^{\infty}\left(\boldsymbol{R}^{n}\right)$ and its dual $L^{\infty *}\left(\boldsymbol{R}^{n}\right)$, which includes $L^{1}\left(\boldsymbol{R}^{n}\right)$. We will denote the natural bilinear pairing between $L^{\infty}\left(\boldsymbol{R}^{n}\right)$ and $L^{\infty *}\left(\boldsymbol{R}^{n}\right)$ by $\langle\tau, \nu\rangle$ for $\tau \in$ $L^{\infty *}\left(\boldsymbol{R}^{n}\right), \nu \in L^{\infty}\left(\boldsymbol{R}^{n}\right)$. In particular, for $\sigma \in L^{1}\left(\boldsymbol{R}^{n}\right)$ and $\nu \in L^{\infty}\left(\boldsymbol{R}^{n}\right)$ we have

$$
\langle\sigma, \nu\rangle=\int_{\boldsymbol{R}^{n}} \sigma(x) \nu(x) d x .
$$

We now define an information state process $\sigma_{k}^{\mu, \epsilon} \epsilon$ $L^{\infty *}\left(\boldsymbol{R}^{n}\right)$ by

$$
\left\langle\sigma_{k}^{\mu, \epsilon}, \eta\right\rangle=\boldsymbol{E}^{\dagger}\left[\eta\left(x_{k}^{\epsilon}\right) \exp \left(\frac{\mu}{\epsilon} \sum_{l=0}^{k-1} L\left(x_{l}^{\epsilon}, u_{l}\right)\right) Z_{k}^{\epsilon} \mid \mathcal{Y}_{k}\right]
$$

for all test functions $\eta$ in $L^{\infty}\left(\boldsymbol{R}^{n}\right)$, for $k=1, \cdots, M$ and $\sigma_{0}^{\mu, \epsilon}=\rho \in L^{1}\left(\boldsymbol{R}^{n}\right)$. We introduce the bounded linear operator $\Sigma^{\mu, \epsilon}: L^{\infty}\left(\boldsymbol{R}^{n}\right) \rightarrow L^{\infty}\left(\boldsymbol{R}^{n}\right)$ defined by

$$
\begin{aligned}
& \Sigma^{\mu \epsilon}(u, y) \nu(\xi) \triangleq \int_{\boldsymbol{R}^{n}} \psi^{\epsilon}(z-b(\xi, u)) \nu(z) d z \\
& \cdot \exp \left(\frac{\mu}{\epsilon} L(\xi, u)\right) \Psi^{\epsilon}(\xi, y) .
\end{aligned}
$$


The bounded linear operator $\Sigma^{\mu, \epsilon^{*}}: L^{\infty *}\left(\boldsymbol{R}^{n}\right) \rightarrow L^{\infty *}\left(\boldsymbol{R}^{n}\right)$ adjoint to $\Sigma^{\mu, \epsilon}$ is defined by

$$
\left\langle\Sigma^{\mu, \epsilon^{*}} \tau, \eta\right\rangle=\left\langle\tau, \Sigma^{\mu, \epsilon} \eta\right\rangle
$$

for all $\tau \in L^{\infty *}\left(\boldsymbol{R}^{n}\right), \eta \in L^{\infty}\left(\boldsymbol{R}^{n}\right)$.

The following theorem establishes that $\sigma_{k}^{\mu, \epsilon}$ is in $L^{1}\left(R^{n}\right)$, and its evolution is governed by the operator $\Sigma^{\mu, \epsilon^{*}}$; for $\sigma \in L^{1}\left(\boldsymbol{R}^{n}\right), \eta \in L^{\infty}\left(\boldsymbol{R}^{n}\right)$, we have

$$
\begin{aligned}
\Sigma^{\mu, \epsilon^{*}}(u, y) \sigma(z)= & \int_{\boldsymbol{R}^{n}} \psi^{\epsilon}(z-b(\xi, u)) \\
& \cdot \exp \left(\frac{\mu}{\epsilon} L(\xi, u)\right) \Psi^{\epsilon}(\xi, y) \sigma(\xi) d \xi .
\end{aligned}
$$

Theorem 2.2: The information state $\sigma_{k}^{\mu, \epsilon}$ satisfies the recursion

$$
\left\{\begin{array}{l}
\sigma_{k}^{\mu, \epsilon}=\Sigma^{\mu, \epsilon^{*}}\left(u_{k-1}, y_{k}^{\epsilon}\right) \sigma_{k-1}^{\mu, \epsilon} \\
\sigma_{0}^{\mu, \epsilon}=\rho
\end{array}\right.
$$

Further, $\sigma_{k}^{\mu, \epsilon} \in L^{1}\left(\boldsymbol{R}^{n}\right)$ since $\rho \in L^{1}\left(\boldsymbol{R}^{n}\right)$ and $\Sigma^{\mu, \epsilon^{*}}$ maps $L^{1}\left(\boldsymbol{R}^{n}\right)$ into $L^{1}\left(\boldsymbol{R}^{n}\right)$.

Proof: We follow Elliott and Moore [11]. From (2.4), we have

$$
\begin{aligned}
\left\langle\sigma_{k}^{\mu, \epsilon}, \eta\right\rangle & \\
= & E^{\dagger}\left[\eta\left(b\left(x_{k-1}^{\epsilon}, u_{k-1}\right)+w_{k-1}^{\epsilon}\right)\right. \\
& \cdot \exp \frac{\mu}{\epsilon} L\left(x_{k-1}^{\epsilon}, u_{k-1}\right) \Psi^{\epsilon}\left(x_{k-1}^{\epsilon}, y_{k}^{\epsilon}\right) \\
& \left.\cdot \exp \frac{\mu}{\epsilon} \sum_{l=0}^{k-2} L\left(x_{l}^{\epsilon}, u_{l}\right) Z_{k-1}^{\epsilon} \mid \mathcal{Y}_{k}\right] \\
= & E^{\dagger}\left[\int_{R^{n}} \eta\left(b\left(x_{k-1}^{\epsilon}, u_{k-1}\right)+w\right)\right. \\
& \cdot \exp \frac{\mu}{\epsilon} L\left(x_{k-1}^{\epsilon}, u_{k-1}\right) \Psi^{\epsilon}\left(x_{k-1}^{\epsilon}, y_{k}^{\epsilon}\right) \\
& \left.\cdot \exp \frac{\mu}{\epsilon} \sum_{l=0}^{k-2} L\left(x_{l}^{\epsilon}, u_{l}\right) Z_{k-1}^{\epsilon} \psi^{\epsilon}(w) d w \mid \mathcal{Y}_{k}\right] \\
= & \left\langle\sigma_{k-1}^{\mu, \epsilon}, \int_{R^{n}} \eta\left(b\left(\cdot, u_{k-1}\right)+w\right)\right. \\
& \left.\cdot \exp \frac{\mu}{\epsilon} L\left(\cdot, u_{k-1}\right) \Psi^{\epsilon}\left(\cdot, y_{k}^{\epsilon}\right) \psi^{\epsilon}(w) d w\right\rangle \\
= & \left\langle\sigma_{k-1}^{\mu, \epsilon}, \Sigma^{\mu, \epsilon}\left(u_{k-1}, y_{k}^{\epsilon}\right) \eta\right\rangle \\
= & \left\langle\Sigma^{\mu, \epsilon^{*}}\left(u_{k-1}, y_{k}^{\epsilon}\right) \sigma_{k-1}^{\mu, \epsilon}, \eta\right\rangle .
\end{aligned}
$$

This holds for all $\eta$ in $L^{\infty}\left(\boldsymbol{R}^{n}\right)$; hence (2.7).

The fact that $\Sigma^{\mu, \epsilon^{*}}$ maps $L^{1}\left(\boldsymbol{R}^{n}\right)$ into $L^{1}\left(\boldsymbol{R}^{n}\right)$ follows easily from (2.6) and the properties of $\psi^{\epsilon}, \Psi^{\epsilon}$, and $L$.

Remark 2.3: When $L \equiv 0$, the recursion (2.7) reduces to the Duncan-Mortensen-Zakai equation for the unnormalized conditional density [22].

The operator $\Sigma^{\mu, \epsilon}$ actually maps $C_{b}\left(\boldsymbol{R}^{n}\right)$ into $C_{b}\left(\boldsymbol{R}^{n}\right)$. Then we can define a process $\nu_{k}^{\mu, \epsilon} \in C_{b}\left(\boldsymbol{R}^{n}\right)$ by

$$
\left\{\begin{array}{l}
\nu_{k-1}^{\mu, \epsilon}=\Sigma^{\mu, \epsilon}\left(u_{k-1}, y_{k}^{\epsilon}\right) \nu_{k}^{\mu, \epsilon} \\
\nu_{M}^{\mu, \epsilon}=\exp \frac{\mu}{\epsilon} \Phi .
\end{array}\right.
$$

It is straightforward to establish the adjoint relationships

$$
\begin{aligned}
\left\langle\Sigma^{\mu, \epsilon^{*}} \sigma, \nu\right\rangle & =\left\langle\sigma, \Sigma^{\mu, \epsilon} \nu\right\rangle \\
\left\langle\sigma_{k}^{\mu, \epsilon}, \nu_{k}^{\mu, \epsilon}\right\rangle & =\left\langle\sigma_{k-1}^{\mu, \epsilon}, \nu_{k-1}^{\mu, \epsilon}\right\rangle
\end{aligned}
$$

for all $\sigma \in L^{1}\left(\boldsymbol{R}^{n}\right), \nu \in C_{b}\left(\boldsymbol{R}^{n}\right)$, and all $k$.

\section{Alternate Representation of the Cost}

Following [4], we define for $u \in \mathcal{U}_{0, M-1}$

$$
K^{\mu, \epsilon}(u)=E^{\dagger}\left[\left\langle\sigma_{M}^{\mu, \epsilon}, \exp \frac{\mu}{\epsilon} \Phi\right\rangle\right]
$$

a cost function associated with the new "state" process $\sigma_{k}^{\mu, \epsilon}$. Theorem 2.4: We have for all $u \in \mathcal{U}_{0, M-1}$

$$
J^{\mu, \epsilon}(u)=K^{\mu, \epsilon}(u)
$$

Proof: By (2.4),

$$
\begin{aligned}
K^{\mu, \epsilon}(u)= & \boldsymbol{E}^{\dagger}\left[\boldsymbol { E } ^ { \dagger } \left[\exp \frac{\mu}{\epsilon} \Phi\left(x_{M}^{\epsilon}\right)\right.\right. \\
& \left.\left.\cdot \exp \frac{\mu}{\epsilon}\left(\sum_{l=0}^{M-1} L\left(x_{l}^{\epsilon}, u_{l}\right)\right) Z_{M}^{\epsilon} \mid \mathcal{Y}_{M}\right]\right] \\
= & \boldsymbol{E}^{\dagger}\left[\exp \frac{\mu}{\epsilon}\left(\sum_{l=0}^{M-1} L\left(x_{l}^{\epsilon}, u_{l}\right)+\Phi\left(x_{M}^{\epsilon}\right)\right) Z_{M}^{\epsilon}\right] \\
= & J^{\mu, \epsilon}(u)
\end{aligned}
$$

using (2.3).

We now define an alternate but equivalent stochastic control problem with complete state information. Under the measure $\boldsymbol{P}^{u}$, consider the state process $\sigma_{k}^{\mu, \epsilon}$ governed by (2.7) and the cost $K^{\mu, \epsilon}(u)$ given by (2.10). The new problem is to find $u^{*} \in \mathcal{U}_{0, M-1}$ minimizing $K^{\mu, \epsilon}$.

Let $\mathcal{U}_{k, l}^{s}$ denote the set of control processes defined on the interval $k, \cdots, l$ which are adapted to $\sigma\left(\sigma_{j}^{\mu, \epsilon}, k \leq j \leq l\right)$. Such policies are called separated [22].

\section{E. Dynamic Programming}

The alternate stochastic control problem can be solved using dynamic programming. Consider now the state $\sigma^{\mu, \epsilon}$ on the interval $k, \cdots, M$ with initial condition $\sigma_{k}^{\mu, \epsilon}=\sigma \in L^{1}\left(\boldsymbol{R}^{n}\right)$ :

$$
\left\{\begin{array}{l}
\sigma_{l}^{\mu, \epsilon}=\Sigma^{\mu, \epsilon^{*}}\left(u_{l-1}, y_{l}^{\epsilon}\right) \sigma_{l-1}^{\mu, \epsilon}, k+1 \leq l \leq M, \\
\sigma_{k}^{\mu, \epsilon}=\sigma .
\end{array}\right.
$$

The corresponding value function for this control problem is defined for $\sigma \in L^{1}\left(R^{n}\right)$ by

$$
S^{\mu, \epsilon}(\sigma, k)=\inf _{u \in \mathcal{U}_{k, M-1}} \boldsymbol{E}^{\dagger}\left[\left\langle\sigma_{k}^{\mu, \epsilon}, \nu_{k}^{\mu, \epsilon}\right\rangle \mid \sigma_{k}^{\mu, \epsilon}=\sigma\right]
$$

Note that this function is expressed in terms of the adjoint process $\nu_{k}^{\mu, \epsilon}$, given by (2.8). 
Theorem 2.5 (Dynamic Programming Equation): The value function $S^{\mu, \epsilon}$ satisfies the recursion

$$
\left\{\begin{array}{l}
S^{\mu, \epsilon}(\sigma, k)=\inf _{u \in U} \boldsymbol{E}^{\dagger}\left[S^{\mu, \epsilon}\left(\Sigma^{\mu, \epsilon^{*}}\left(u, y_{k+1}^{\epsilon}\right) \sigma, k+1\right)\right] \\
S^{\mu, \epsilon}(\sigma, M)=\left\langle\sigma, \exp \frac{\mu}{\epsilon} \Phi\right\rangle .
\end{array}\right.
$$

Proof: The proof is similar to Elliott and Moore [11, Theorem 4.5].

$$
\begin{aligned}
& S^{\mu, \epsilon}(\sigma, k) \inf _{u \in \mathcal{U}_{k, k}} \inf _{v \in \mathcal{U}_{k+1, M-1}} \\
& \boldsymbol{E}^{\dagger}\left[\left\langle\sigma_{k}^{\mu, \epsilon}, \Sigma^{\mu, \epsilon}\left(u_{k}, y_{k+1}^{\epsilon}\right) \nu_{k+1}^{\mu, \epsilon}\right\rangle \mid \sigma_{k}^{\mu, \epsilon}=\sigma\right] \\
&=\inf _{u \in \mathcal{U}_{k, k}} \inf _{v \in \mathcal{U}_{k+1, M-1}} \\
& \boldsymbol{E}^{\dagger}\left[\boldsymbol{E}^{\dagger}\left[\left\langle\Sigma^{\mu, \epsilon^{*}}\left(u_{k}, y_{k+1}^{\epsilon}\right) \sigma_{k}^{\mu, \epsilon}, \nu_{k+1}^{\mu, \epsilon}\right\rangle \mid \mathcal{Y}_{k+1}\right] \mid\right. \\
&\left.=\sigma_{k}^{\mu, \epsilon}=\sigma\right] \\
& \inf _{u \in \mathcal{U}_{k, k}} \boldsymbol{E}^{\dagger}\left[\inf _{v \in \mathcal{U}_{k+1, M-1}}\right. \\
& \boldsymbol{E}^{\dagger}\left[\left\langle\Sigma^{\mu, \epsilon^{*}}\left(u_{k}, y_{k+1}^{\epsilon}\right) \sigma_{k}^{\mu, \epsilon}, \nu_{k+1}^{\mu, \epsilon}\right\rangle \mid \mathcal{Y}_{k+1}\right] \mid \\
&\left.=\sigma_{k}^{\mu, \epsilon}=\sigma\right] \\
& \inf _{u \in \mathcal{U}_{k, k}} \boldsymbol{E}^{\dagger}\left[\inf _{v \in \mathcal{U}_{k+1, M-1}}\right. \\
&\left.\boldsymbol{E}^{\dagger}\left[\left\langle\sigma_{k+1}^{\mu, \epsilon}, \nu_{k+1}^{\mu, \epsilon}\right\rangle \mid \sigma_{k+1}^{\mu, \epsilon}=\Sigma^{\mu, \epsilon^{*}}\left(u_{k}, y_{k+1}^{\epsilon}\right) \sigma\right]\right] \\
&=\inf _{u \in \mathcal{U}_{k, k}} \boldsymbol{E}^{\dagger}\left[S^{\mu, \epsilon}\left(\Sigma^{\mu, \epsilon^{*}}\left(u_{k}, y_{k+1}^{\epsilon}\right) \sigma, k+1\right)\right]
\end{aligned}
$$

The interchange of minimization and conditional expectation is justified because of the lattice property of the set of controls [9, Chapter 16].

Theorem 2.6 (Verification): Suppose that $u^{*} \in \mathcal{U}_{0, M-1}^{s}$ is a policy such that, for each $k=0, \cdots, M-1, u_{k}^{*}=\bar{u}_{k}^{*}\left(\sigma_{k}^{\mu, \epsilon}\right)$, where $\bar{u}_{k}^{*}(\sigma)$ achieves the minimum in (2.14). Then $u^{*} \in$ $\mathcal{U}_{0, M-1}$ and is an optimal policy for the partially observed risk-sensitive stochastic control problem (Section II-B).

Proof: We follow the proof of Elliott and Moore [11, Theorem 4.7]. Define

$$
\bar{S}^{\mu, \epsilon}(\sigma, k ; u)=E^{\dagger}\left[\left\langle\sigma_{k}^{\mu, \epsilon}, \nu_{k}^{\mu, \epsilon}\right\rangle \mid \sigma_{k}^{\mu, \epsilon}=\sigma\right] .
$$

We claim that

$$
S^{\mu, \epsilon}(\sigma, k)=\bar{S}^{\mu, \epsilon}\left(\sigma, k ; u^{*}\right)
$$

for each $k=0,1, \cdots, M$.

For $k=M,(2.15)$ is clearly satisfied. Assume now that (2.15) holds for $k+1, \cdots, M$. Then

$$
\begin{aligned}
\bar{S}^{\mu, \epsilon}\left(\sigma, k ; u^{*}\right)= & \boldsymbol{E}^{\dagger}\left[\boldsymbol { E } ^ { \dagger } \left[\left\langle\Sigma^{\mu, \epsilon^{*}}\left(u_{k}^{*}, y_{k+1}^{\epsilon}\right) \sigma_{k}^{\mu, \epsilon}\right.\right.\right. \\
& \left.\left.\left.\cdot \nu_{k+1}^{\mu, \epsilon}\left(u_{k+1, M-1}^{*}\right)\right\rangle \mid \mathcal{Y}_{k+1}\right] \mid \sigma_{k}^{\mu, \epsilon}=\sigma\right] \\
= & \boldsymbol{E}^{\dagger}\left[\overline { S } ^ { \mu , \epsilon } \left(\Sigma^{\mu, \epsilon^{*}}\left(u_{k}^{*}, y_{k+1}^{\epsilon}\right) \sigma,\right.\right. \\
& \left.\left.\cdot k+1 ; u_{k+1, M-1}^{*}\right)\right] \\
= & \boldsymbol{E}^{\dagger}\left[S^{\mu, \epsilon}\left(\Sigma^{\mu, \epsilon^{*}}\left(u_{k}^{*}, y_{k+1}^{\epsilon}\right) \sigma, k+1\right)\right] \\
= & S^{\mu, \epsilon}(\sigma, k)
\end{aligned}
$$

from (2.14). This proves (2.15).
From (2.15), setting $k=0$ and $\sigma=\rho \in L^{1}\left(\boldsymbol{R}^{n}\right)$ we obtain

$$
\bar{S}^{\mu, \epsilon}\left(\rho, 0 ; u^{*}\right)=S^{\mu, \epsilon}(\rho, 0) \leq \bar{S}^{\mu, \epsilon}(\rho, 0 ; u)
$$

for any $u \in \mathcal{U}_{0, M-1}$. Comparing (2.10) and the definitions of $S^{\mu, \epsilon}, \bar{S}^{\mu, \epsilon}$ above this implies

$$
K^{\mu, \epsilon}\left(u^{*}\right) \leq K^{\mu, \epsilon}(u)
$$

for all $u \in \mathcal{U}_{0, M-1}$. Using Theorem 2.4, we complete the proof.

Remark 2.7: The significance of Theorem 2.6 is that it establishes the optimal policy of the risk-sensitive stochastic control problem as a separated policy through the process $\sigma_{k}^{\mu, \epsilon}$, defined in Section II-C, which serves as an "information state" [22]. The information state $\sigma_{k}^{\mu, \epsilon}$ is a sufficient statistic for the risk-sensitive problem [25].

\section{SMALl NOISE LIMIT}

To obtain a limit variational problem as $\epsilon \rightarrow 0$, we must first obtain limit results for the information state (and its dual).

\section{A. Information State}

For $\gamma \in G \triangleq\left\{\gamma \in \boldsymbol{R}^{2}: \gamma_{1}>0, \gamma_{2} \geq 0\right\}$ define

$$
\mathcal{D}^{\gamma} \triangleq\left\{p \in C\left(\boldsymbol{R}^{n}\right): p(x) \leq-\gamma_{1}|x|^{2}+\gamma_{2}\right\}
$$

$\mathcal{D} \triangleq\left\{p \in C\left(\boldsymbol{R}^{n}\right): p(x) \leq-\gamma_{1}|x|^{2}+\gamma_{2}\right.$ for some $\left.\gamma \in G\right\}$

and write

$$
C_{b}\left(\boldsymbol{R}^{n}\right) \triangleq\left\{q \in C\left(\boldsymbol{R}^{n}\right):|q(x)| \leq C, \text { for some } C \geq 0\right\} .
$$

We equip these spaces with the topology of uniform convergence on compact subsets. In the sequel, $B(x, \alpha) \subset \boldsymbol{R}^{p}$ denotes the open ball centered at $x \in R^{p}$ of radius $\alpha>0$.

The "sup pairing"

$$
(p, q) \triangleq \sup _{x \in \boldsymbol{R}^{n}}\{p(x)+q(x)\}
$$

is defined for $p \in \mathcal{D}, q \in C_{b}\left(\boldsymbol{R}^{n}\right)$ and arises naturally in view of the Varadhan-Laplace Lemma (see the Appendix):

$$
\lim _{\epsilon \rightarrow 0} \frac{\epsilon}{\mu} \log \left\langle e^{\mu / \epsilon p}, e^{\mu / \epsilon q}\right\rangle=(p, q)
$$

(uniformly on compact subsets of $\mathcal{D}^{\gamma} \times C_{b}\left(\boldsymbol{R}^{n}\right)$, for each $\gamma \in G)$.

Define operators $\Lambda^{\mu *}: \mathcal{D} \rightarrow \mathcal{D}$, and $\Lambda^{\mu}: C_{b}\left(\Re^{n}\right) \rightarrow$ $C_{b}\left(\Re^{n}\right)$ by

$$
\begin{aligned}
\Lambda^{\mu *}(u, y) p(z) \triangleq & \sup _{\xi \in \boldsymbol{R}^{n}}\left\{L(\xi, u)-\frac{1}{2 \mu}|z-b(\xi, u)|^{2}\right. \\
& \left.-\frac{1}{\mu}\left[\frac{1}{2}|h(\xi)|^{2}-h(\xi) y\right]+p(\xi)\right\} \\
\Lambda^{\mu}(u, y) q(\xi) \triangleq & \sup _{z \in R^{n}}\left\{-\frac{1}{2 \mu}|z-b(\xi, u)|^{2}+q(z)\right\} \\
& +L(\xi, u)-\frac{1}{\mu}\left[\frac{1}{2}|h(\xi)|^{2}-h(\xi) y\right] .
\end{aligned}
$$


With respect to the "sup pairing" $(\cdot, \cdot)$, these operators satisfy

$$
\left(\Lambda^{\mu *} p, q\right)=\left(p, \Lambda^{\mu} q\right) \text {. }
$$

Also, $\Lambda^{\mu *}(u, y): \mathcal{D}^{\gamma} \rightarrow \mathcal{D}$ is continuous for each $\gamma \in G$; in fact, the map $(u, y, p) \mapsto \Lambda^{\mu *}(u, y) p, U \times \boldsymbol{R} \times \mathcal{D}^{\gamma} \rightarrow \mathcal{D}$ is continuous.

The next theorem is a logarithmic limit result for the information state and its dual, stated in terms of operators (i.e., semigroups).

Theorem 3.1: We have

$$
\begin{aligned}
\lim _{\epsilon \rightarrow 0} \frac{\epsilon}{\mu} \log \Sigma^{\mu, \epsilon^{*}}(u, y) e^{\mu / \epsilon p} & =\Lambda^{\mu *}(u, y) p, \\
\lim _{\epsilon \rightarrow 0} \frac{\epsilon}{\mu} \log \Sigma^{\mu, \epsilon}(u, y) e^{\mu / \epsilon q} & =\Lambda^{\mu}(u, y) q
\end{aligned}
$$

in $\mathcal{D}$ uniformly on compact subsets of $U \times \boldsymbol{R} \times \mathcal{D}^{\gamma}$ for each $\gamma \in G$, respectively in $C_{b}\left(\boldsymbol{R}^{n}\right)$ uniformly on compact subsets of $U \times \boldsymbol{R} \times C_{b}\left(\boldsymbol{R}^{n}\right)$.

Proof: From (2.6), we have

$$
\begin{aligned}
\frac{\epsilon}{\mu} \log \Sigma^{\mu, \epsilon^{*}}(u, y) e^{\mu / \epsilon p}(z) \\
=\frac{\epsilon}{\mu} \log \int_{R^{n}} \exp \frac{\mu}{\epsilon}\left(-\frac{1}{2 \mu}|z-b(\xi, u)|^{2}-\frac{n \epsilon}{2 \mu} \log (2 \pi \epsilon)\right. \\
\left.\quad-\frac{1}{\mu}\left[\frac{1}{2}|h(\xi)|^{2}-h(\xi) y\right]+L(\xi, u)+p(\xi)\right) d \xi
\end{aligned}
$$

Therefore,

$$
\begin{aligned}
\lim _{\epsilon \rightarrow 0} \frac{\epsilon}{\mu} \log \Sigma^{\mu, \epsilon^{*}}(u, y) e^{\mu / \epsilon p}(z) \\
=\sup _{\xi \in \boldsymbol{R}^{n}}\left\{L(\xi, u)-\frac{1}{2 \mu}|z-b(\xi, u)|^{2}\right. \\
\left.\quad-\frac{1}{\mu}\left[\frac{1}{2}|h(\xi)|^{2}-h(\xi) y\right]+p(\xi)\right\} \\
=\Lambda^{\mu *}(u, y) p(z)
\end{aligned}
$$

uniformly in $a=(z, u, y, p) \in A$, by Lemma A.1; where $A=B(0, R) \times U \times B(0, R) \times K$, and $K \subset \mathcal{D}^{\gamma}$ is compact. This proves the first part of (3.5). The second part is proven similarly.

\section{B. Risk-Sensitive Value Function}

The next theorem evaluates the small noise limit of the risksensitive value function $S^{\mu, \epsilon}$. It involves two large deviations type limits, one corresponding to estimation and one to control.

Theorem 3.2: The function $W^{\mu}(p, k)$ defined for $p \in \mathcal{D}$ by

$$
W^{\mu}(p, k) \triangleq \lim _{\epsilon \rightarrow 0} \frac{\epsilon}{\mu} \log S^{\mu, \epsilon}\left(e^{\mu / \epsilon p}, k\right)
$$

exists (i.e., the sequence converges uniformly on compact subsets of $\mathcal{D}^{\gamma}(\gamma \in G)$ ), is continuous on $\mathcal{D}^{\gamma}(\gamma \in G)$, and satisfies the recursion

$$
\left\{\begin{array}{c}
W^{\mu}(p, k)=\inf _{u \in U} \sup _{y \in \boldsymbol{R}} \\
\left\{W^{\mu}\left(\Lambda^{\mu *}(u, y) p, k+1\right)-\frac{1}{2 \mu}|y|^{2}\right\} \\
W^{\mu}(p, M)=(p, \Phi)
\end{array}\right.
$$

Proof: The result is clearly true for $k=M$ because of the second of (2.14), (3.2), and the continuity of $p \mapsto(p, \Phi)$ on each $\mathcal{D}^{\gamma}$.

Assume the conclusions hold for $k+1, \cdots, M$. Select $\gamma \in G$ and $K \subset \mathcal{D}^{\gamma}$ compact. In what follows $C>0$, etc, will denote a universal constant. From Theorem 2.5 and (3.6), we need to compute

$$
\begin{aligned}
\lim _{\epsilon \rightarrow 0} \frac{\epsilon}{\mu} \log S^{\mu, \epsilon}\left(e^{\mu / \epsilon p}, k\right) \\
=\lim _{\epsilon \rightarrow 0} \frac{\epsilon}{\mu} \log \inf _{u \in U} E^{\dagger}\left[S ^ { \mu , \epsilon } \left(\Sigma^{\mu, \epsilon^{*}}\left(u, y_{k+1}^{\epsilon}\right)\right.\right. \\
\left.\left.\quad \cdot e^{\mu / \epsilon p}, k+1\right)\right] \\
=\lim _{\epsilon \rightarrow 0} \inf _{u \in U} \frac{\epsilon}{\mu} \log E^{\dagger}\left[S ^ { \mu , \epsilon } \left(\Sigma^{\mu, \epsilon^{*}}\left(u, y_{k+1}^{\epsilon}\right)\right.\right. \\
\left.\left.\quad \cdot e^{\mu / \epsilon p}, k+1\right)\right] .
\end{aligned}
$$

The last equality is due to the monotonicity of the logarithm function.

Direct calculation verifies the estimate

$$
\frac{\epsilon}{\mu} \log S^{\mu, \epsilon}\left(\Sigma^{\mu, \epsilon^{*}}(u, y) e^{\mu / \epsilon p}, k+1\right) \leq C(1+|y|)
$$

for all $u \in U, y \in \boldsymbol{R}, p \in K, \epsilon<\epsilon^{\prime}$ for some $\epsilon^{\prime}>0$, and the inclusion

$$
\frac{\epsilon}{\mu} \log \Sigma^{\mu, \epsilon^{*}}(u, y) e^{\mu / \epsilon p} \in \mathcal{D}^{\gamma(|y|)}
$$

for all $u \in U, y \in \boldsymbol{R}, p \in K, \epsilon<\epsilon^{\prime}$, for some $\gamma(|y|) \in G$. The fact that $\gamma(|y|)$ depends on $|y|$ complicates matters a little. If we select $R>0$ and consider those $y$ for which $|y| \leq R$, then there exists $\gamma_{R} \in G$ such that

$$
\frac{\epsilon}{\mu} \log \Sigma^{\mu, \epsilon^{*}}(u, y) e^{\mu / \epsilon p} \in \mathcal{D}^{\gamma_{R}}
$$

for all $u \in U,|y| \leq R, p \in K$, for all $\epsilon>0$ sufficiently small. Considering the right-hand side of (3.8) we have

$$
\begin{aligned}
& V^{\mu, \epsilon}(p, k ; u) \\
& \triangleq \frac{\epsilon}{\mu} \log \boldsymbol{E}^{\dagger}\left[S^{\mu, \epsilon}\left(\Sigma^{\mu, \epsilon^{*}}\left(u, y_{k+1}^{\epsilon}\right) e^{\mu / \epsilon p}, k+1\right)\right] \\
& =\frac{\epsilon}{\mu} \log \int_{\boldsymbol{R}} \phi^{\epsilon}(y) S^{\mu, \epsilon}\left(\Sigma^{\mu, \epsilon^{*}}(u, y) e^{\mu / \epsilon p}, k+1\right) d y \\
& =\frac{\epsilon}{\mu} \log \left\{\int_{|y| \leq R} S^{\mu, \epsilon}\left(\Sigma^{\mu, \epsilon^{*}}(u, y) e^{\mu / \epsilon p}, k+1\right) d y\right. \\
& \left.\quad+\int_{|y| \geq R} \phi^{\epsilon}(y) S^{\mu, \epsilon}\left(\Sigma^{\mu, \epsilon^{*}}(u, y) e^{\mu / \epsilon p}, k+1\right) d y\right\} \\
& \triangleq \\
& \frac{\epsilon}{\mu} \log \{A+B\} .
\end{aligned}
$$

Now using the bounds above we can write

$$
\begin{array}{r}
\frac{\epsilon}{\mu} \log B \leq \frac{\epsilon}{\mu} \log \int_{|y| \geq R} \exp \frac{\mu}{\epsilon}\left(C(1+|y|)-\frac{1}{2 \mu}|y|^{2}\right. \\
\left.-\frac{\epsilon}{2 \mu} \log (2 \pi \epsilon)\right) d y
\end{array}
$$

and using a standard estimate for Gaussian integrals we obtain

$$
\begin{aligned}
\frac{\epsilon}{\mu} \log B & \leq C_{1}-C_{2} R^{2} \\
& \leq-C^{\prime}
\end{aligned}
$$


as $\epsilon \rightarrow 0$, uniformly in $u \in U, p \in K$, where $C^{\prime}>0$ if $R>0$ is chosen sufficiently large.

By the induction hypothesis $W^{\mu}(p, k+1)$ exists and

$$
W^{\mu}(p, k+1)=\lim _{\epsilon \rightarrow 0} \frac{\epsilon}{\mu} \log S^{\mu, \epsilon}\left(e^{\mu / \epsilon p}, k+1\right)
$$

uniformly on $\mathcal{D}^{\gamma_{R}}$. We also have from Theorem 3.1 that

$$
\Lambda^{\mu *}(u, y) p=\lim _{\epsilon \rightarrow 0} \frac{\epsilon}{\mu} \log \Sigma^{\mu, \epsilon^{*}}(u, y) e^{\mu / \epsilon p}
$$

uniformly on $U \times B(0, R) \times K$, and

$$
\Lambda^{\mu *}(u, y) p \in \mathcal{D}^{\gamma_{R}}
$$

for all $(u, y, p) \in U \times B(0, R) \times K$.

Consider the function $W^{\mu}\left(\Lambda^{\mu *}(u, y) p, k+1\right)-1 / 2 \mu|y|^{2}$ Due to the induction hypothesis and the properties of $\Lambda^{\mu, \epsilon}$, it is continuous in $p, y, u$, and bounded in $y$; all properties uniformly in $(u, p) \in U \times K$. Therefore we can choose $R$ large enough so that enough so that both $(3.10)$ is satisfied and

$$
\underset{y \in R}{\operatorname{argmax}}\left\{W^{\mu}\left(\Lambda^{\mu *}(u, y) p, k+1\right)-\frac{1}{2 \mu}|y|^{2}\right\} \subset B(0, R) .
$$

We keep $R$ fixed from now on.

Combining the above limits for $S^{\mu, \epsilon}$ and $\Sigma^{\mu, \epsilon}$ we have that

$$
\begin{aligned}
\lim _{\epsilon \rightarrow 0} & \frac{\epsilon}{\mu} \log \left\{S^{\mu, \epsilon}\left(\Sigma^{\mu, \epsilon^{*}}(u, y) e^{\mu / \epsilon p}, k+1\right)\right. \\
\cdot & \left.\exp \frac{\mu}{\epsilon}\left(-\frac{1}{2 \mu}|y|^{2}-\frac{\epsilon}{2 \mu} \log (2 \pi \epsilon)\right)\right\} \\
= & W^{\mu}\left(\Lambda^{\mu *}(u, y) p, k+1\right)-\frac{1}{2 \mu}|y|^{2}
\end{aligned}
$$

uniformly in $u \in U, p \in K, y \in B(0, R)$.

We can now proceed with the further computation of (3.9). Indeed, we follow the proof of Lemma A.1 with

$$
\begin{array}{r}
S^{\mu, \epsilon}\left(\Sigma^{\mu, \epsilon^{*}}(u, y) e^{\mu / \epsilon p}, k+1\right) \\
\cdot \exp \frac{\mu}{\epsilon}\left(-\frac{1}{2 \mu}|y|^{2}-\frac{\epsilon}{2 \mu} \log (2 \pi \epsilon)\right)
\end{array}
$$

replacing $\exp \left(F_{a}^{\epsilon} / \epsilon\right)$, and

$$
W^{\mu}\left(\Lambda^{\mu *}(u, y) p, k+1\right)-\frac{1}{2 \mu}|y|^{2}
$$

replacing $F_{a}$, and $a=(u, p) \in A=U \times K$. Then

$$
\lim _{\epsilon \rightarrow 0} \frac{\epsilon}{\mu} \log A=\sup _{y \in R}\left\{W^{\mu}\left(\Lambda^{\mu *}(u, y) p, k+1\right)-\frac{1}{2 \mu}|y|^{2}\right\}
$$

uniformly in $U \times K$. It is crucial that $R$ is chosen as above so that the uniform bound required by condition iii) of Lemma A.1 is satisfied. Consequently

$$
\begin{aligned}
& \lim _{\epsilon \rightarrow 0} V^{\mu, \epsilon}(p, k ; u) \\
& =\lim _{\epsilon \rightarrow 0} \frac{\epsilon}{\mu} \log (A+B) \\
& =\lim _{\epsilon \rightarrow 0} \frac{\epsilon}{\mu} \log A(1+B / A)
\end{aligned}
$$

$$
\begin{aligned}
& =\lim _{\epsilon \rightarrow 0} \frac{\epsilon}{\mu} \log A \\
& =\sup _{y \in \boldsymbol{R}}\left\{W^{\mu}\left(\Lambda^{\mu *}(u, y) p, k+1\right)-\frac{1}{2 \mu}|y|^{2}\right\}
\end{aligned}
$$

uniformly on $U \times K$, since $\lim _{\epsilon \rightarrow 0} \epsilon / \mu \log (1+B / A)=0$ from (3.10) and (3.12).

To complete the proof, we use the continuity of the map $(p, u) \mapsto V^{\mu, \epsilon}(p, k ; u), \mathcal{D}^{\gamma} \times U \rightarrow \boldsymbol{R}$ to obtain

$$
\begin{aligned}
& \lim _{\epsilon \rightarrow 0} \frac{\epsilon}{\mu} \log S^{\mu, \epsilon}\left(e^{\mu / \epsilon p}, k\right) \\
& =\lim _{\epsilon \rightarrow 0} \inf _{u \in U} V^{\mu, \epsilon}(p, k ; u) \\
& =\inf _{u \in U} \lim _{\epsilon \rightarrow 0} V^{\mu, \epsilon}(p, k ; u) \\
& =\inf _{u \in U} \sup _{y \in \boldsymbol{R}}\left\{W^{\mu}\left(\Lambda^{\mu *}(u, y) p, k+1\right)-\frac{1}{2 \mu}|y|^{2}\right\} \\
& =W^{\mu}(p, k)
\end{aligned}
$$

uniformly on $K$. The last equality holds by the definition (3.6).

The sequence converges uniformly on $K$, and as a result $W^{\mu}(p, k)$ is continuous on $\mathcal{D}^{\gamma}$. This completes the proof.

Remark 3.3: In Section IV (3.7) will be interpreted as the optimal cost function (upper value) for a dynamic game problem.

Remark 3.4: Note that there are two large deviation type limits involved in the result of Theorem 3.2. The first is expressed in the use of (3.5) in (3.11) and corresponds to "state estimation" or "observers." The second is embodied in (3.6) and corresponds to the relationship of the stochastic risksensitive optimal control problem to the deterministic game that (3.7), (3.13) imply.

\section{A Dynamic Game Problem}

\section{A. Dynamics}

We consider a two-player deterministic partially observed dynamic game for the discrete-time system

$$
\left\{\begin{array}{l}
x_{k+1}=b\left(x_{k}, u_{k}\right)+w_{k} \\
y_{k+1}=h\left(x_{k}\right)+v_{k}
\end{array}\right.
$$

on the finite time interval $k=0,1,2, \cdots M$, where

i) $x(0)=x_{0}$ is the unknown initial condition, and $y(0)=$ 0 .

ii) Player 1 selects the $U$-valued control $u_{k}$, which is required to be a nonanticipating functional of the observation path $y$. We write $\mathcal{U}_{k, l}$ for the set of such controls defined on the interval $k, \cdots, l$, and note that $u \in \mathcal{U}_{k, l}$ if and only if for each $j \in[k, l]$ there exists a function $\bar{u}_{j}: \boldsymbol{R}^{(j-k+1) n} \rightarrow U$ such that $u_{j}=\bar{u}_{j}\left(y_{k+1, j}\right)$.

iii) Player 2 selects the $\boldsymbol{R}^{n} \times \boldsymbol{R}$-valued disturbance $\left(w_{k}, v_{k}\right)$, which is a square summable open-loop sequence. We let $l_{2}\left([k, l], \boldsymbol{R}^{p}\right)$ denote the set of square summable $\boldsymbol{R}^{p}$-valued sequences defined on the interval $k, \cdots, l(p=n, 1, n+1)$. 


\section{B. Cost}

The payoff function for the game is defined for admissible $u \in \mathcal{U}_{0, M-1}$ and $(w, v) \in l_{2}\left([0, M-1], \boldsymbol{R}^{n+1}\right)$ by

$$
\begin{array}{r}
J^{\mu}(u, w, v)=\sup _{x_{0} \in \boldsymbol{R}^{n}}\left\{\alpha\left(x_{0}\right)+\sum_{l=0}^{M-1} L\left(x_{l}, u_{l}\right)+\Phi\left(x_{M}\right)\right. \\
\left.-\frac{1}{\mu} \sum_{l=0}^{M-1} \frac{1}{2}\left(\left|w_{l}\right|^{2}+\left|v_{l}\right|^{2}\right)\right\},
\end{array}
$$

where $\alpha \in \mathcal{D}$.

Remark 4.1: This formulation treats $x_{0}$ as part of the uncertainty (to be chosen by nature). A priori knowledge of $x_{0}$ is incorporated in the cost function $\alpha \in \mathcal{D}$. Our theory also applies to the case $\alpha=0$, which corresponds to no a priori information, and note $\lim _{\epsilon \rightarrow 0} \epsilon \log \rho=0$, where $\rho$ is the initial density for the risk-sensitive stochastic control problem. One can alternatively select $\alpha \in \mathcal{D}$ and define the initial density by $\rho(x)=c_{\epsilon} \exp (-\alpha(x) / \epsilon)$, where $c_{\epsilon}$ is a normalizing constant.

The (upper) game is defined as follows. Let

$$
J^{\mu}(u)=\sup _{(w, v) \in l_{2}\left([0, M-1], \boldsymbol{R}^{n+1}\right)} J^{\mu}(u, w, v),
$$

and the (upper) partially observed dynamic game problem is to find $u^{*} \in \mathcal{U}_{0, M-1}$ such that

$$
J^{\mu}\left(u^{*}\right)^{\prime}=\inf _{u \in \mathcal{U}_{0, M-1}} J^{\mu}(u)
$$

The cost function $J^{\mu}(u)$ can be rewritten in the form

$$
\begin{aligned}
J^{\mu}(u)= & \sup _{x \in l_{2}\left([0, M], \boldsymbol{R}^{n}\right) y \in l_{2}([1, M], \boldsymbol{R})} \sup ^{M-1} L\left(\alpha\left(x_{0}\right)+\sum_{l=0}^{M-1} L\left(x_{l}, u_{l}\right)+\Phi\left(x_{M}\right)-\frac{1}{\mu} \sum_{l=0}^{M-1} \frac{1}{2}\right. \\
& \left.\cdot\left(\left|x_{l+1}-b\left(x_{l}, u_{l}\right)\right|^{2}+\left|y_{l+1}-h\left(x_{l}\right)\right|^{2}\right)\right\}
\end{aligned}
$$

This cost function is finite for all $\mu>0$.

\section{Information State}

Motivated by the use of an information state and associated separation policies in solving the risk-sensitive stochastic control problem, given a control policy $u \in \mathcal{U}_{0, M-1}$ and an observation path $y \in l_{2}([0, M], \boldsymbol{R})$, we define an "information state" $p_{k}^{\mu} \in \mathcal{D}$ and its "dual" $q_{k}^{\mu} \in C_{b}\left(\boldsymbol{R}^{n}\right)$ for the game problem by the recursions

$$
\begin{aligned}
& \left\{\begin{array}{l}
p_{k}^{\mu}=\Lambda^{\mu *}\left(u_{k-1}, y_{k}\right) p_{k-1}^{\mu} \\
p_{0}^{\mu}=\alpha
\end{array}\right. \\
& \left\{\begin{array}{l}
q_{k-1}^{\mu}=\Lambda^{\mu}\left(u_{k-1}, y_{k}\right) q_{k}^{\mu} \\
q_{M}^{\mu}=\Phi
\end{array}\right.
\end{aligned}
$$

where $\Lambda^{\mu}$ and $\Lambda^{\mu *}$ are as defined in (3.3). Note that, in the "sup pairing" notation of (3.1),

$$
\left(p_{k}^{\mu}, q_{k}^{\mu}\right)=\left(p_{k-1}^{\mu}, q_{k-1}^{\mu}\right)
$$

for all $k$, and

$$
\sigma_{k}^{\mu, \epsilon} \sim \exp \frac{\mu}{\epsilon} p_{k}^{\mu}, \quad \nu_{k}^{\mu, \epsilon} \sim \exp \frac{\mu}{\epsilon} q_{k}^{\mu}
$$

in probability as $\epsilon \rightarrow 0$.

Remark 4.2: The asymptotic formulas (4.7) are similar to the large deviation limit for nonlinear filtering ([15], [16], [19], [20]). In addition, if $L \equiv 0$ the recursions (4.4) reduce to the equations for Mortensen's method of minimum energy estimation.

\section{Alternate Representation of the Cost}

Define for $u \in \mathcal{U}_{0, M-1}$

$K^{\mu}(u)=\sup _{y \in l_{2}([1, M], R)}\left\{\left(p_{M}^{\mu}, \Phi\right)-\frac{1}{2 \mu} \sum_{l=0}^{M-1}\left|y_{l+1}\right|^{2}\right\}$

a cost function associated with the new "state" process $p_{k}^{\mu}$.

Theorem 4.3: We have for all $u \in \mathcal{U}_{0, M-1}$

$$
J^{\mu}(u)=K^{\mu}(u) .
$$

Proof: Iterating (4.4), we see that

$$
\begin{aligned}
p_{M}^{\mu}(z)= & \sup _{x \in l_{2}\left([0, M], \boldsymbol{R}^{n}\right)}\left\{\alpha\left(x_{0}\right)+\sum_{l=0}^{M-1} L\left(x_{l}, u_{l}\right)\right. \\
& -\frac{1}{\mu} \sum_{l=0}^{M-1} \frac{1}{2}\left|x_{l+1}-b\left(x_{l}, u_{l}\right)\right|^{2} \\
& \left.-\frac{1}{\mu} \sum_{l=0}^{M-1}\left[\frac{1}{2}\left|h\left(x_{l}\right)\right|^{2}-h\left(x_{l}\right) y_{l+1}\right]: x_{M}=z\right\} .
\end{aligned}
$$

Substitution of this equality into (4.8) yields (4.9).

We now define an alternate deterministic dynamic game problem with complete state information. Consider the state sequence $p_{k}^{\mu}$ with dynamics (4.4) and the cost $K^{\mu}(u)$ : find $u^{*} \in \mathcal{U}_{0, M-1}^{s}$ minimizing $K^{\mu}$. Here, $\mathcal{U}_{k, l}^{s}$ denotes the set of separated control policies, through the information state $p_{k}^{\mu}$, defined on the interval $k, \cdots, l$, i.e., those which are nonanticipating functionals of $\left(p_{j}^{\mu}, k \leq j \leq l\right)$. Note that $\mathcal{U}_{k, l}^{s} \subset \mathcal{U}_{k, l}$.

\section{E. Dynamic Programming}

Consider now the state $p^{\mu}$ on the interval $k, \cdots, M$ with initial condition $p_{k}^{\mu}=p \in \mathcal{D}$

$$
\left\{\begin{array}{l}
p_{l}^{\mu}=\Lambda^{\mu *}\left(u_{l-1}, y_{l}\right) p_{l-1}^{\mu}, \quad k+1 \leq l \leq M, \\
p_{k}^{\mu}=p .
\end{array}\right.
$$

The (upper) value function is defined for $p \in \mathcal{D}$ by

$$
\begin{aligned}
W^{\mu}(p, k)= & \left.\inf _{u \in \mathcal{U}_{k, M-1}} \sup _{y \in l_{2}([k+1, M]}, \boldsymbol{R}\right) \\
& \left\{\left(p_{k}^{\mu}, q_{k}^{\mu}\right)-\frac{1}{2 \mu} \sum_{l=k}^{M-1}\left|y_{l+1}\right|^{2}: p_{k}^{\mu}=p\right\} .
\end{aligned}
$$

Theorem 4.4: The value function $W^{\mu}(p, k)$ defined by (4.11) is the unique solution of the dynamic programming equation (3.7). 
Proof: From (4.11), (4.4), (4.5), and (4.6), we have

$$
\begin{aligned}
& W^{\mu}(p, k)=\inf _{\boldsymbol{u}_{k} \in \mathcal{U}_{k, k}} \inf _{v \in \mathcal{U}_{k+1, M-1}} \sup _{y_{k+1} \in \boldsymbol{R}_{\boldsymbol{y}} \in l_{2}([k+2, M), \boldsymbol{R})} \sup \\
& \cdot\left\{\left(p_{k}^{\mu}, \Lambda^{\mu}\left(u_{k}, y_{k+1}\right) q_{k+1}^{\mu}\left(v_{k+1, M-1}\right)\right)\right. \\
& \left.-\frac{1}{2 \mu} \sum_{l=k+1}^{M-1}\left|y_{l+1}\right|^{2}-\frac{1}{2 \mu}\left|y_{k+1}\right|^{2}: p_{k}^{\mu}=p\right\}
\end{aligned}
$$

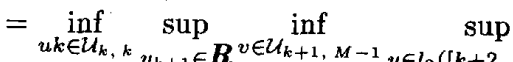

$$
\begin{aligned}
& \cdot\left\{\left(\Lambda^{\mu *}\left(u_{k}, y_{k+1}\right) p_{k}^{\mu}, q_{k+1}^{\mu}\left(v_{k+1, M-1}\right)\right)\right. \\
& \left.-\frac{1}{2 \mu} \sum_{l=k+1}^{M-1}\left|y_{l+1}\right|^{2}-\frac{1}{2 \mu}\left|y_{k+1}\right|^{2}: p_{k}^{\mu}=p\right\} \\
& =\inf _{u_{k} \in \mathcal{U}_{k, k}} \sup _{y_{k+1} \in \boldsymbol{R}}\left\{W^{\mu}\left(\Lambda^{\mu *}\left(u_{k}, y_{k+1}\right) p, k+1\right)\right. \\
& \left.-\frac{1}{2 \mu}\left|y_{k+1}\right|^{2}\right\}
\end{aligned}
$$

which is the same as (3.7). Here, the interchange of the minimization over $v_{k+1, M-1}$ and maximimization over $y_{k+1}$ is justified because $v_{k+1, M-1}$ is a function of $y_{k+1}$.

Remark 4.5: We conclude from Theorem 3.2 and 4.4 that the small noise limit of the partially observed stochastic risksensitive problem is a partially observed deterministic dynamic game problem.

Theorem 4.6 (Verification): Suppose that $u^{*} \in \mathcal{U}_{0, M-1}^{s}$ is a policy such that, for each $k=0, \cdots, M-1, u_{k}^{*}=\bar{u}_{k}^{*}\left(p_{k}^{\mu}\right)$, where $\bar{u}_{k}^{*}(p)$ achieves the minimum in (3.7). Then $u^{*} \in$ $\mathcal{U}_{0, M-1}$ and is an optimal policy for the partially observed dynamic game problem (Sections IV-A, IV-B)

Proof: Define

$$
\begin{aligned}
& W^{\mu}(p, k ; u) \\
& =\sup _{y \in l_{2}([k+1, M], R)}\left\{\left(p_{k}^{\mu}, q_{k}^{\mu}\right)-\frac{1}{2 \mu} \sum_{l=k}^{M-1}\left|y_{l+1}\right|^{2}: p_{k}^{\mu}=p\right\} .
\end{aligned}
$$

We claim that

$$
W^{\mu}(p, k)=W^{\mu}\left(p, k ; u^{*}\right)
$$

for each $k=0,1, \cdots, M$. For $k=M,(4.12)$ is true. Assume now that (4.12) holds for $k+1, \cdots, M$. Then

$$
\begin{aligned}
& W^{\mu}\left(p, k ; u^{*}\right) \\
& =\sup _{y_{k+1} \in \boldsymbol{R}_{y \in l_{2}([k+2, M], \boldsymbol{R})}} \sup \\
& \quad \cdot\left\{\left(\Lambda^{\mu *}\left(u_{k}^{*}, y_{k+1}\right) p_{k}^{\mu}, q_{k+1}^{\mu}\left(u^{*} k+1, M-1\right)\right)\right. \\
& \left.\quad-\frac{1}{2 \mu} \sum_{l=k+1}^{M-1}\left|y_{l+1}\right|^{2}-\frac{1}{2 \mu}\left|y_{k+1}\right|^{2}: p_{k}^{\mu}=p\right\} \\
& =\sup _{y_{k+1} \in \boldsymbol{R}}\left\{W^{\mu}\left(\Lambda^{\mu *}\left(u_{k}^{*}, y_{k+1}\right) p_{k}^{\mu}, k+1 ; u_{k+1, M-1}^{*}\right)\right.
\end{aligned}
$$

$$
\begin{aligned}
& \left.-\frac{1}{2 \mu}\left|y_{k+1}\right|^{2}: p_{k}^{\mu}=p\right\} \\
= & \sup _{y_{k+1} \in \boldsymbol{R}}\left\{W^{\mu}\left(\Lambda^{\mu *}\left(u_{k}^{*}, y_{k+1}\right) p, k+1\right)-\frac{1}{2 \mu}\left|y_{k+1}\right|^{2}\right\} \\
= & W^{\mu}(p, k)
\end{aligned}
$$

which proves (4.12).

Next, from (4.12) and setting $k=0$ and $p=\alpha$ we obtain

$$
W^{\mu}\left(\alpha, 0 ; u^{*}\right)=W^{\mu}(\alpha, 0) \leq W^{\mu}(\alpha, 0 ; u)
$$

for all $u \in \mathcal{U}_{0, M-1}$, which implies

$$
K^{\mu}\left(u^{*}\right) \leq K^{\mu}(u)
$$

for all $u \in U_{0, M-1}$. This together with Theorem 4.3 completes the proof.

\section{F. Certainty Equivalence Principle}

Remark 4.7: Theorem 4.6 is the "appropriate" separation theorem for the partially observed dynamic game described in Sections IV-A and IV-B, in that it establishes that the optimal feedback policy is a separated one [22] through the information state $p_{k}^{\mu}$ which carries all the information from the observations $y_{0, k}$ relevant for control. It is important to note that the solution of this partially observed dynamic game problem involves two infinite dimensional recursions. One is (4.4), which describes the dynamics of the information state, evolves forward in time, and is a dynamic programming equation in view of (3.3). This equation plays the role of an "observer" in the resulting controller and is determined by the control problem at hand, and not prescribed a priori. The information state $p_{k}^{\mu}(x)$ is a function of the state variable $x \in \boldsymbol{R}^{n}$. The other recursion is (3.7), which describes the computation of the feedback control as a function of the information state, evolves backward in time, and is a dynamic programming equation. The value function $W^{\mu}(p, k)$ is a function of the information state variable $p$, which takes values in the infinitedimensional space $\mathcal{D}$. An important aspect of our work is to point out this essential difficulty of the nonlinear robust $H_{\infty}$ control problem. This is not surprising given that this difficulty is well known in stochastic control. For practical applications, one can try to find suboptimal finite-dimensional schemes that provide performance close to the one predicted by the optimal results obtained here. We are also pursuing the development of numerical schemes that can compute the required recursions (4.4) and (3.7), as well as the incorporation of such schemes into computer-aided design control systems design software based on optimization.

We now relate the above analysis to certainty equivalence principles suggested by Whittle [26], [28] for the risk-sensitive problem, and by Basar and Bernhard [3] for the game problem.

Consider a completely observed dynamic game problem with dynamics

$$
x_{k+1}=b\left(x_{k}, u_{k}\right)+w_{k}
$$

and payoff function

$$
J^{\mu}(u, w)=\sum_{l=0}^{M-1} L\left(x_{l}, u_{l}\right)+\Phi\left(x_{M}\right)-\frac{1}{\mu} \sum_{l=0}^{M-1} \frac{1}{2}\left|w_{l}\right|^{2}
$$


where the initial state $x_{0}$ is known, player 1 selects $u \in$ $\mathcal{U}_{0, M-1}^{c}$ to minimize $J^{\mu}$, and player 2 selects $w \in l_{2}([0, M-$ $1), \boldsymbol{R}^{n}$ ) to maximize $J^{\mu}$. Here, $\mathcal{U}_{k, l}^{c}$ is the set of $U$-valued controls which are nonanticipating functionals of the state $x$ defined on the interval $k, \cdots, l$.

Define the upper value (see e.g., [10]) for this dynamic game by

$$
\begin{array}{r}
\bar{f}_{k}^{\mu}(x)=\inf _{u \in \mathcal{U}_{k, M-1}^{c},} \sup _{w \in l_{2}\left([k, M-1], \boldsymbol{R}^{n}\right)}\left\{\sum_{l=k}^{M-1} L\left(x_{l}, u_{l}\right)\right. \\
\left.+\Phi\left(x_{M}\right)-\frac{1}{\mu} \sum_{l=k}^{M-1} \frac{1}{2}\left|w_{l}\right|^{2}: x_{k}=x\right\} .
\end{array}
$$

This function satisfies the dynamic programming equation

$$
\left\{\begin{array}{c}
\bar{f}_{k}^{\mu}(x)=\inf _{u \in U} \sup _{w \in \boldsymbol{R}^{n}} \\
\left\{\bar{f}_{k+1}^{\mu}(b(x, u)+w)+L(x, u)-\frac{1}{2 \mu}|w|^{2}\right\} \\
\bar{f}_{M}^{\mu}(x)=\Phi(x)
\end{array}\right.
$$

and if $\bar{u}_{k}^{*}(x) \in U$ achieves the minimum in (4.16), then $u_{k}^{*}=\bar{u}_{k}^{*}\left(x_{k}\right)$ is an optimal feedback policy for this completely observed game.

Whittle [26], [28] solves the partially observed risk-sensitive stochastic control problem by using the solution to the completely observed game (4.16) and the modified filter or "observer" (4.4). He refers to $\bar{f}_{k}^{\mu}$ as the future stress, to $p_{k}^{\mu}$ as the past stress, and defines the minimum stress estimate $\bar{x}_{k}$ of $x_{k}$ by

$$
\bar{x}_{k} \in \underset{x \in \boldsymbol{R}^{n}}{\operatorname{argmax}}\left\{p_{k}^{\mu}(x)+\bar{f}_{k}^{\mu}(x)\right\} \triangleq \hat{x}_{k}^{\mu}
$$

where $\hat{x}_{k}^{\mu}$ is a set-valued variable. For linear systems with the quadratic cost, the certainty equivalence principle asserts that if $J^{\mu}(u, w, v)$ is negative definite in $(w, v)$ (and positive definite in $u$ ), then $u_{k}^{*}=\bar{v}_{k}^{*}\left(\bar{x}_{k}\right)$ is an optimal control for the partially observed risk-sensitive problem [26]. For nonlinear systems, Whittle's assertion is that this recipe gives a policy which is approximately optimal for the risk-sensitive stochastic control problem [20].

Remark 4.8: The variable $\hat{x}_{k}^{\mu}$ is set-valued and is closely related to the finite-time observer results of James [19, (3:8)] and our earlier observer design methodology [1], [20]. Indeed our construction brings out another essential difficulty of the nonlinear problem, which has to do with multivalued (or setvalued) variables for state estimation and control. We will have more to say about this issue in a forthcoming paper.

We now state a certainty equivalence principle for the partially observed deterministic game problem described in $[3$, Chapters 5, 6].

Theorem 4.9 (Certainty Equivalence): Let $\bar{f}_{k}^{\mu}(x)$ be the upper value for the full state information game (4.13), (4.14). If for all $k=0, \cdots, M$ and $p \in \mathcal{D}$ we have

$$
W^{\mu}(p, k)=\left(p, \bar{f}_{k}^{\mu}\right)
$$

then the policy $u^{c e} \in \mathcal{U}_{0, M-1}^{s}$ defined by

$$
u_{k}^{c e}=\tilde{u}_{k}^{*}\left(\vec{x}_{k}\right)
$$

is an optimal policy for the partially observed game problem (Sections IV-A and IV-B).

Proof: Let

$$
x_{k}^{*}(p) \in \underset{\xi \in \boldsymbol{R}^{n}}{\operatorname{argmax}}\left(p(\xi)+\bar{f}_{k}^{\mu}(\xi)\right) .
$$

Then the minimum stress estimate and the candidate policy defined by (4.19) can be written as

$$
\bar{x}_{k}=x_{k}^{*}\left(p_{k}^{\mu}\right) \quad \text { and } \quad u_{k}^{c e}=\tilde{u}_{k}^{*}\left(x_{k}^{*}\left(p_{k}^{\mu}\right)\right) .
$$

Therefore $u_{k}^{c e}$ is a separated policy. To check the optimality of $u_{k}^{c e}$, we apply the verification Theorem 4.6. We must show that for each $k, \bar{u}_{k}^{c e}(p) \triangleq \tilde{u}_{k}^{*}\left(x_{k}^{*}(p)\right)$ achieves the minimum in (3.7). To prove this, using the hypothesis (4.18) at time $k+1$, we have

$$
\begin{aligned}
W^{\mu}(p, k) & \inf _{u \in U} \sup _{y \in \boldsymbol{R}}\left\{W^{\mu}\left(\Lambda^{\mu *}(u, y) p, k+1\right)-\frac{1}{2 \mu}|y|^{2}\right\} \\
= & \inf _{u \in U} \sup _{y \in \boldsymbol{R}}\left\{\left(\Lambda^{\mu *}(u, y) p, \bar{f}_{k+1}^{\mu}\right)-\frac{1}{2 \mu}|y|^{2}\right\} \\
= & \inf _{u \in U} \sup _{y \in \boldsymbol{R}} \sup _{x \in \boldsymbol{R}^{n}} \sup _{\xi \in \boldsymbol{R}^{n}} \\
& \cdot\left\{p(\xi)+L(\xi, u)-\frac{1}{2 \mu}|z-b(\xi, u)|^{2}\right. \\
& \left.-\frac{1}{\mu}\left[\frac{1}{2}|h(\xi)|^{2}-h(\xi) y\right]-\frac{1}{2 \mu}|y|^{2}+\bar{f}_{k+1}^{\mu}(z)\right\} \\
= & \inf _{u \in U} \sup _{\xi \in \boldsymbol{R}^{n}}(p(\xi)+L(\xi, u) \\
& \left.+\sup _{z \in \boldsymbol{R}^{n}}\left\{-\frac{1}{2 \mu}|z-b(\xi, u)|^{2}+\bar{f}_{k+1}^{\mu}(z)\right\}\right) .
\end{aligned}
$$

On the other hand, (4.18) at time $k$ implies

$$
\begin{aligned}
W^{\mu}(p, k)= & \sup _{\xi \in \boldsymbol{R}^{n}}\left(p(\xi)+\bar{f}_{k}^{\mu}(\xi)\right) \\
= & \sup _{\xi \in \boldsymbol{R}^{n}} \inf _{u \in U}(p(\xi)+L(\xi, u) \\
& \left.+\sup _{z \in \boldsymbol{R}^{n}}\left\{-\frac{1}{2 \mu}|z-b(\xi, u)|^{2}+\bar{f}_{k+1}^{\mu}(z)\right\}\right) .
\end{aligned}
$$

Thus $W^{\mu}(p, k)$ is a saddle value for a static game, with saddle point $\xi=x_{k}^{*}(p), u=\tilde{u}_{k}^{*}\left(x_{k}^{*}(p)\right)$. Therefore $\tilde{u}_{k}^{*}\left(x_{k}^{*}(p)\right)$ achieves the minimum in (3.7).

Remark 4.10: As described in Remark 4.7, the partially observed dynamic game (and the related robust control problem [2]) involve two infinite-dimensional recursions. The significance of the "certainty equivalence" theorem is that if valid, the recursion (3.7) involving the value $W^{\mu}(p, k), p \in \mathcal{D}$ can be replaced by a simpler recursion (4.16) involving the upper value $\bar{f}_{k}^{\mu}(x), x \in \boldsymbol{R}^{n}$. This has obvious computational implications. 
Remark 4.11: A crucial contribution here is that we have identified precisely the condition that one needs to establish the certainty equivalence principle suggested in [3], [26], [28]. The condition is $(4.18)$, i.e., the saddle point condition in (4.21), (4.22). One may ask to what extent this condition can be obviated. Or alternatively, can we show under certain assumptions that (4.18) is satisfied? A counterexample to the certainty equivalence principle is given in Bernhardsson [5], and hence this principle is not valid in general. Indeed, (4.18) may not hold in general, and the proof of Theorem 4.9 shows that

$$
W^{\mu}(p, k) \geq\left(p, \bar{f}_{k}^{\mu}\right)
$$

This is not surprising from the point of view of stochastic control, since the certainty equivalence principle is not valid generally in that context.

Remark 4.12: Since the partially observed game is the limit of the partially observed risk-sensitive problem, then, if the certainty equivalence principle is valid, the policy (4.19) is an approximate optimal policy for the partially observed risksensitive problem for small $\epsilon>0$ [28].

Remark 4.13: The Bertsekas and Rhodes paper [6] considers game problems similar to the type discussed here. In the language of that paper, the information state $p_{k}^{\mu}$ is a sufficiently informative function, i.e., the deterministic dynamic game analogue of sufficient statistic [25].

\section{SMALL RISK LIMIT}

In this section we show that a risk-neutral stochastic control problem is obtained if in the risk-sensitive stochastic control problem the risk-sensitivity parameter $\mu$ tends to zero.

\section{A. Information State} by

Define the bounded linear operator $\Sigma^{\epsilon^{*}}: L^{1}\left(\boldsymbol{R}^{n}\right) \rightarrow L^{1}\left(\boldsymbol{R}^{n}\right)$

$$
\Sigma^{\epsilon^{*}}(u, y) \sigma(z) \triangleq \int_{\boldsymbol{R}^{n}} \psi^{\epsilon}(z-b(\xi, u)) \Psi^{\epsilon}(\xi, y) \sigma(\xi) d \xi
$$

Theorem 5.1: We have

$$
\lim _{\mu \rightarrow 0} \Sigma^{\mu, \epsilon^{*}}(u, y) \sigma=\Sigma^{\epsilon^{*}}(u, y) \sigma
$$

uniformly on bounded subsets of $U \times \boldsymbol{R} \times L^{1}\left(\boldsymbol{R}^{n}\right)$.

Proof: This result follows simply from the definitions (2.6), (5.1).

Next, we define a process $\sigma_{k}^{\epsilon} \in L^{1}\left(\boldsymbol{R}^{n}\right)$ by the recursion

$$
\left\{\begin{array}{l}
\sigma_{k}^{\epsilon}=\sum^{\epsilon *}\left(u_{k-1}, y_{k}^{\epsilon}\right) \sigma_{k-1}^{\epsilon} \\
\sigma_{0}^{\epsilon}=\rho
\end{array}\right.
$$

Remark 5.2: The process $\sigma_{k}^{\epsilon}$ is an unnormalized conditional density of $x_{k}^{\epsilon}$ given $\mathcal{Y}_{k}$, and (5.3) is known as the Duncan-Mortensen-Zakai equation [11], [22].

\section{B. A Risk-Neutral Control Problem}

We again consider the discrete-time stochastic system (2.1) and formulate a partially observed risk-neutral stochastic control problem with cost

$$
J^{\epsilon}(u)=E^{u}\left[\sum_{l=0}^{M-1} L\left(x_{l}^{\epsilon}, u_{l}\right)+\Phi\left(x_{M}^{\epsilon}\right)\right]
$$

defined for $u \in \mathcal{U}_{0, M-1}$, where $\mathcal{U}_{0, M-1}$, etc., are as defined in Section II. This cost function is finite for all $\epsilon>0$.

We quote the following result from [11], [22], which establishes that the optimal policy is separated through the information state $\sigma_{k}^{\epsilon}$ satisfying (5.3).

Theorem 5.3: The unnormalized conditional density $\sigma_{k}^{\epsilon}$ is an information state for the risk-neutral problem, and the value function defined for $\sigma \in L^{1}\left(\boldsymbol{R}^{n}\right)$ by

$$
\begin{aligned}
W^{\epsilon}(\sigma, k)=\inf _{u \in \mathcal{U}_{k, M-1}} \boldsymbol{E}^{\dagger}\left[\sum_{l=k}^{M-1}\left\langle\sigma_{l}^{\epsilon}, L(\cdot, u)\right\rangle\right. & \\
& \left.+\left\langle\sigma_{M}^{\epsilon}, \Phi\right\rangle \mid \sigma_{k}^{\epsilon}=\sigma\right]
\end{aligned}
$$

satisfies the dynamic programming equation

$$
\left\{\begin{array}{c}
W^{\epsilon}(\sigma, k)=\inf _{u \in U} \\
\boldsymbol{E}^{\dagger}\left[\langle\sigma, L(\cdot, u)\rangle+W^{\epsilon}\left(\sum^{\epsilon *}\left(u, y_{k+1}^{\epsilon}\right) \sigma, k+1\right)\right] \\
W^{\epsilon}(\sigma, M)=\langle\sigma, \Phi\rangle
\end{array}\right.
$$

If $u^{*} \in \mathcal{U}_{0, M-1}^{s}$ is a policy such that, for each $k=0, \cdots, M-$ $1, u_{k}^{*}=\bar{u}_{k}^{*}\left(\sigma_{k}^{\epsilon}\right)$, where $\bar{u}_{k}^{*}(\sigma)$ achieves the minimum in (5.6), then $u^{*} \in \mathcal{U}_{0, M-1}$ and is an optimal policy for the partially observed risk-neutral problem.

Remark 5.4: The function $W^{\epsilon}(\sigma, k)$ depends continuously on $\sigma \in L^{1}\left(\boldsymbol{R}^{n}\right)$.

\section{Risk-Sensitive Value Function}

The next theorem evaluates the small risk limit of the risksensitive stochastic control problem. Note that normalization of the information state is required.

Theorem 5.5: We have

$$
\lim _{\mu \rightarrow 0} \frac{\epsilon}{\mu} \log \frac{S^{\mu, \epsilon}(\sigma, k)}{\langle\sigma, 1\rangle}=\frac{W^{\epsilon}(\sigma, k)}{\langle\sigma, 1\rangle}
$$

uniformly on bounded subsets of $L^{1}\left(\boldsymbol{R}^{n}\right)$.

Proof: 1) We claim that

$$
S^{\mu, \epsilon}(\sigma, k)=\langle\sigma, 1\rangle+\frac{\mu}{\epsilon} W^{\epsilon}(\sigma, k)+o(\mu)
$$

as $\mu \rightarrow 0$ uniformly on bounded subsets of $L^{1}\left(\boldsymbol{R}^{n}\right)$.

For $k=M$,

$$
\begin{aligned}
S^{\mu, \epsilon}(\sigma, M) & =\left\langle\sigma, e^{\mu / \epsilon \Phi}\right\rangle \\
& =\langle\sigma, 1\rangle+\frac{\mu}{\epsilon}\langle\sigma, \Phi\rangle+o(\mu) \\
& =\langle\sigma, 1\rangle+\frac{\mu}{\epsilon} W^{\epsilon}(\sigma, M)+o(\mu)
\end{aligned}
$$

as $\mu \rightarrow 0$, uniformly on bounded subsets of $L^{1}\left(\boldsymbol{R}^{n}\right)$. 
Assume now that (5.8) is true for $k+1, \cdots, M$. Then

$$
\begin{aligned}
& V^{\mu, \epsilon}(\sigma, k ; u) \\
& \triangleq \boldsymbol{E}^{\dagger}\left[S^{\mu, \epsilon}\left(\Sigma^{\mu, \epsilon^{*}}\left(u, y_{k+1}^{\epsilon}\right) \sigma, k+1\right)\right] \\
& =\int_{\boldsymbol{R}} \phi^{\epsilon}(y) S^{\mu, \epsilon}\left(\Sigma^{\mu, \epsilon^{*}}(u, y) \sigma, k+1\right) d y \\
& =\int_{\boldsymbol{R}^{n}} \int_{\boldsymbol{R}} \phi^{\epsilon}(y-h(\xi)) \exp \frac{\mu}{\epsilon} L(\xi, u) \\
& \cdot S^{\mu, \epsilon}\left(\frac{\Sigma^{\mu, \epsilon^{*}}(u, y) \sigma}{\left\langle\Sigma^{\mu, \epsilon^{*}}(u, y) \sigma, 1\right\rangle}, k+1\right) \sigma(\xi) d \xi d y \\
& =\int_{\boldsymbol{R}^{n}} \int_{\boldsymbol{R}} \phi^{\epsilon}(y-h(\xi))\left\{1+\frac{\mu}{\epsilon} L(\xi, u)+o(\mu)\right\} \\
& \cdot\left\{1+\frac{\mu}{\epsilon} W^{\epsilon}\left(\frac{\Sigma^{\mu, \epsilon^{*}}(u, y) \sigma}{\left\langle\Sigma^{\mu, \epsilon^{*}}(u, y) \sigma, 1\right\rangle}, k+1\right)+o(\mu)\right\} \\
& \cdot \sigma(\xi) d \xi d y \\
& =\langle\sigma, 1\rangle+\frac{\mu}{\epsilon}\{\langle\sigma, L(\cdot, u)\rangle \\
& +\int_{\boldsymbol{R}^{n}} \int_{\boldsymbol{R}} \phi^{\epsilon}(y-h(\xi)) W^{\epsilon}\left(\frac{\sum^{\epsilon *}(u, y) \sigma}{\left\langle\sum^{\epsilon *}(u, y) \sigma, 1\right\rangle}, k+1\right) \\
& \cdot \sigma(\sigma) d \xi d y\}+o(\mu) \\
& =\langle\sigma, 1\rangle+\frac{\mu}{\epsilon}\left\{\langle\sigma, L(\cdot, u)\rangle+\int_{\boldsymbol{R}} \phi^{\epsilon}(y) W^{\epsilon}\right. \\
& \left.\cdot\left(\sum^{\epsilon *}(u, y) \sigma, k+1\right) d y\right\}+o(\mu)
\end{aligned}
$$

as $\mu \rightarrow 0$ uniformly on bounded subsets of $U \times L^{1}\left(\boldsymbol{R}^{n}\right)$. Thus, using the continuity of $(\sigma, \mu) \mapsto V^{\mu, \epsilon}(\sigma, k ; u)$

$$
\begin{aligned}
& S^{\mu, \epsilon}(\sigma, k) \\
& =\inf _{u \in U} V^{\mu \epsilon}(\sigma, k ; u) \\
& =\langle\sigma, 1\rangle+\frac{\mu}{\epsilon} \inf _{u \in U}\{\langle\sigma, L(\cdot, u)\rangle \\
& \left.\quad+\int_{\boldsymbol{R}} \phi^{\epsilon}(y) W^{\epsilon}\left(\sum^{\epsilon *}(u, y) \sigma, k+1\right) d y\right\}+o(\mu) \\
& =\langle\sigma, 1\rangle+\frac{\mu}{\epsilon} W^{\epsilon}(\sigma, k)+o(\mu)
\end{aligned}
$$

uniformly on bounded subsets of $L^{1}\left(\boldsymbol{R}^{n}\right)$, proving (5:8).

2) To complete the proof, note that (5.8) implies

$$
\frac{S^{\mu, \epsilon}(\sigma, k)}{\langle\sigma, 1\rangle}=1+\frac{\mu}{\epsilon} \frac{W^{\epsilon}(\sigma, k)}{\langle\sigma, 1\rangle}+o(\mu)
$$

and hence

$$
\frac{\epsilon}{\mu} \log \frac{S^{\mu, \epsilon}(\sigma, k)}{\langle\sigma, 1\rangle}=\frac{W^{\epsilon}(\sigma, k)}{\langle\sigma, 1\rangle}+o(1)
$$

as $\mu \rightarrow 0$, uniformly on bounded subsets of $L^{1}\left(\boldsymbol{R}^{n}\right)$.

Remark 5.6: We conclude from Theorems 5.3 and 5.5 that the small risk limit of the partially observed stochastic risksensitive problem is a partially observed stochastic risk-neutral problem.

\section{APPENDIX A}

The following theorem is a version of the Varadhan-Laplace Lemma [13]. Below $\varrho$ denotes a metric on $C\left(\boldsymbol{R}^{n}\right)$ corresponding to uniform convergence on compact subsets, and $B(x, \alpha)$ denotes the open ball centered at $x$ of radius $\alpha$.

Lemma A.1: Let $A$ be a compact space, $F_{a}^{\epsilon}, F_{a} \in C\left(\boldsymbol{R}^{m}\right)$ and assume

i)

$$
\lim _{\epsilon \rightarrow 0} \sup _{a \in A} \varrho\left(F_{a}^{\epsilon}, F_{a}\right)=0 .
$$

ii) The function $F_{a}$ is uniformly continuous on each set $B(0, R), R>0$, uniformly in $a \in A$.

iii) There exist $\gamma_{1}>0, \gamma_{2} \geq 0$ such that

$$
F_{a}^{\epsilon}(x), F_{a}(x) \leq-\gamma_{1}|x|^{2}+\gamma_{2}
$$

for all $x \in \boldsymbol{R}^{m}, a \in A, \epsilon>0$.

Then

$$
\lim _{\boldsymbol{\epsilon} \rightarrow 0} \sup _{a \in A}\left|\epsilon \log \int_{\boldsymbol{R}^{n}} e^{F_{a}^{\epsilon}(x) / \epsilon} d x-\sup _{x \in \boldsymbol{R}^{n}} F_{a}(x)\right|=0 .
$$

Proof: Write $\bar{F}_{a}^{\epsilon}=\sup _{x \in R^{m}} F_{a}^{\epsilon}(x), \bar{F}_{a}=$ $\sup _{x \in \boldsymbol{R}^{m}} F_{a}(x)$. Our assumptions ensure that

$$
\lim _{\epsilon \rightarrow 0} \sup _{a \in A} \bar{F}_{a}^{\epsilon}=\bar{F}_{a} \text {. }
$$

For $\delta>0$ define

$$
B_{\delta}^{a, \epsilon}=\left\{x \in R^{m}: F_{a}^{\epsilon}(x)>\bar{F}_{a}^{\epsilon}-\delta\right\} .
$$

Then the uniform coercivity hypothesis iii) ensures there exists $R>0$ such that $B_{\delta}^{a, \epsilon} \subset B(0, R)$.

By hypothesis ii) on $B(0, R)$ and using the uniform convergence on $B(0, R)$, given $\delta>0$ there exists $r>0$ such that

$$
\left|x-x^{\prime}\right|<r \text { implies }\left|F_{a}^{\epsilon}(x)-F_{a}^{\epsilon}\left(x^{\prime}\right)\right|<\delta
$$

for all $x, x^{\prime} \in B(0, R), a \in A$, and $\epsilon>0$ sufficiently small.

Let $x_{a}^{\epsilon} \in \operatorname{argmax} F_{a}^{\epsilon}$. Then $\left|x-x_{a}^{\epsilon}\right|<r$ implies $\mid F_{a}^{\epsilon}(x)-$ $\bar{F}_{a}^{\epsilon} \mid<\delta$ for all $a \in A$, and $\epsilon>0$ sufficiently small. Hence

$$
B\left(x_{a}^{\epsilon}, r\right) \subset B_{\delta}^{a, \epsilon}
$$

for all $a \in A$, and $\epsilon>0$ sufficiently small. Then

$$
\begin{aligned}
\alpha_{a}^{\epsilon} & \triangleq \int_{R^{n}} \exp \left(F_{a}^{\epsilon}(x) / \epsilon\right) d x \\
& \geq \int_{B_{\delta}^{a, e}} \exp \left(F_{a}^{\epsilon}(x) / \epsilon\right) d x \\
& \geq C_{m} r^{m} \exp \left(\frac{\bar{F}_{a}^{\epsilon}-\delta}{\epsilon}\right) .
\end{aligned}
$$

Thus

$$
\begin{aligned}
\epsilon \log \alpha_{a}^{\epsilon} & \geq \epsilon \log C_{m} r^{m}+\bar{F}_{a}^{\epsilon}-\delta \\
& \geq \bar{F}_{a}-3 \delta
\end{aligned}
$$

for all $\epsilon>0$ sufficiently small and all $a \in A$. 


$$
\begin{aligned}
& \text { Next, for } R>0 \text { write } \\
& \begin{aligned}
\alpha_{a}^{\epsilon} & =\int_{|x| \leq R} \exp \left(F_{a}^{\epsilon}(x) / \epsilon\right) d x+\int_{|x| \geq R} \exp \left(F_{a}^{\epsilon}(x) / \epsilon\right) d x \\
& =A+B
\end{aligned}
\end{aligned}
$$

and note that

$$
\epsilon \log \alpha_{a}^{\epsilon}=\epsilon \log A+O(B / A) .
$$

Now

$$
\begin{aligned}
B & \leq \int_{|x| \geq R} \exp \left(\frac{-\gamma_{1}|x|^{2}+\gamma_{2}}{\epsilon}\right) d x \\
& \leq C_{R} \exp \left(\frac{C_{1}-C_{2} R^{2}}{\epsilon}\right) \\
& \leq C_{R} \exp \left(-C^{\prime} / \epsilon\right)
\end{aligned}
$$

where $C_{R}, C_{1}, C_{2}>0$, and $C^{\prime}>0$ if $R$ is chosen sufficiently large. Also

$$
\begin{aligned}
\epsilon \log A & \leq \epsilon \log \int_{|x| \leq R} \exp \left(\bar{F}_{a}^{\epsilon} / \epsilon\right) d x \\
& \leq \epsilon \log C_{m} R^{m}+\bar{F}_{a}^{\epsilon}
\end{aligned}
$$

where $R$ is chosen large enough to ensure that $\operatorname{argmax}_{x \in \boldsymbol{R}^{m}} F_{a}^{\epsilon}(x) \subset B(0, R)$ for all $a \in A$ and all sufficiently small $\epsilon$. Thus

$$
\epsilon \log \alpha_{a}^{\epsilon} \leq \bar{F}_{a}+3 \delta
$$

for all $\epsilon>0$ sufficiently small and all $a \in A$.

Combining (A.2) and (A.3) we obtain

$$
\sup _{a \in A}\left|\epsilon \log \alpha_{a}^{\epsilon}-\bar{F}_{a}\right|<3 \delta
$$

for all $\epsilon>0$ sufficiently small. This proves the lemma.

\section{REFERENCES}

[1] J. S. Baras, A. Bensoussan, and M. R. James, "Dynamic observers as asymptotic limits of recursive filters: Special cases," SIAM J. Applied Math., vol. 48, no. 5, pp. 1147-1158, 1988.

[2] J. S. Baras and M. R. James, "Robust output feedback control for nonlinear discrete-time systems, preprint, 1993.

[3] T. Basar and P. Bernhard, " $H^{\infty}$-Optimal Control and Related Minimax Design Problems: A Dynamic Game Approach" Boston: Birkhauser, 1991.

[4] A. Bensoussan and J. H. van Schuppen, "Optimal control of partially observable stochastic systems with an exponential-of-integral performance index," SIAM J. Contr. Optim., vol. 23, pp. 599-613, 1985.

[5] B. Bernhardsson, "Topics in digital and robust control of linear systems," Ph.D. dissertation, Lund Institute of Technology, Lund, Sweden, 1992.

[6] D. P. Bertsekas and I. B. Rhodes, "Sufficiently informative functions and the minimax feedback control of uncertain dynamical systems," IEEE Trans. Automat. Contr., vol. 18, pp. 117-123, 1973.

[7] M. Campi and M. R. James, "Discrete-time nonlinear risk-sensitive control," preprint, 1992.

[8] J. C. Doyle, K. Glover, P. P. Khargonekar, and B. A. Francis, "Statespace solutions to standard $\mathrm{H}_{2}$ and $\mathrm{H}_{\infty}$ control problems," IEEE Trans. Automat. Contr., vol. 34, no. 8, pp. 831-847, 1989.

[9] R. J. Elliott, Stochastic Calculus and Applications. New York: Springer-Verlag, 1982.

[10] Scientific and Technical, Pitman Research Notes in Mathematics Series, 1987.

[11] R. J. Elliott and J. B. Moore, "Discrete time partially observed control," 12th IFAC World Congress, Sydney, 1993.
[12] W. H. Fleming and W. M. McEneaney, "Risk sensitive optimal control and differential games," in Proc. Conf. Adaptive Stochastic Contr., Univ. of Kansas, 1991.

[13] M. I. Freidlin and A. D. Wentzell, Random Perturbations of Dynamical Systems. New York: Springer-Verlag, 1984.

[14] K. Glover and J. C. Doyle, "State space formulae for all stabilizing controllers that satisfy an $H_{\infty}$ norm bound and relations to risk sensitivity," Syst. Contr. Lett., vol. 11, pp. 167-172, 1988.

[15] O. Hijab, "Minimum energy estimation," Ph.D. dissertation, Univ. of Calif., Berkeley, 1980.

[16] _ "Asmptotic Bayesian estimation of a first order equation with a small diffusion," Annals of Prob., vol. 12, pp. 890-902, 1984.

[17] D. H. Jacobson, "Optimal stochastic linear systems with exponential performance criteria and their relation to deterministic differential games," IEEE Trans. Automat. Contr., vol. AC-18, no. 2, pp. 124-131, 1973.

[18] M. R. James, "Asymptotic analysis of nonlinear stochastic risk-sensitive control and differential games," Math. Contr. Sign. Syst., vol. 5, no. 4, pp. $401-417,1992$.

[19] 2 "Finite time observer design by probabilistic-variational methods, SIAM J. Contr. Optim., vol. 29, no. 4, pp. 954-967, 1991.

[20] M. R. James and J. S. Baras, "Nonlinear filtering and large deviations: A PDE-control theoretic approach," Stochastics, vol. 23, no. 3, pp. $391-412,1988$.

[21] M. R. James, J. S. Baras, and R. J. Elliott, "Output feedback risksensitive control and differential games for continuous-time nonlinear systems," 32nd IEEE CDC 1993.

[22] P. R. Kumar and P. Varaiya, Stochastic Systems: Estimation, Identification, and Adaptive Control. Englewood Cliffs, NJ: Prentice-Hall, 1986.

[23] D. J. N. Limebeer, B. D. O. Anderson, P. P. Khargonekar, and M. Green, "A game theoretic approach to $H_{\infty}$ control for time varying systems," SIAM J. Contr. Optim., vol. 30, pp. 262-283, 1992.

[24] I. Rhee and J. L. Speyer, "A game theoretic approach to a finite-time disturbance attenuation problem, IEEE Trans. Automat. Contr., yol. 36, pp. 1021-1032, 1991.

[25] C. Striebel, Optimal Control of Discrete Time Stochastic Systems (Lecture Notes in Economics and Mathematical Systems). Berlin: SpringerVerlag, 1975

[26] P. Whittle, "Risk-sensitive linear/quadratic/gaussian control," Adv. Appl. Prob., vol. 13, pp. 764-777, 1981

[27] _ , "A risk-sensitive maximum principle," Syst. Contr. Lett., vol. 15, pp. 183-192, 1990.

[28] - "A risk-sensitive maximum principle: The case of imperfect state observation, IEEE Trans. Automat. Control, vol. 36, pp. 793-801, 1991.

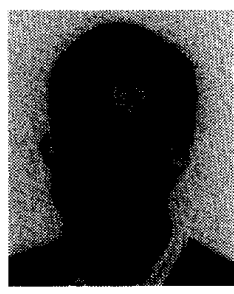

Matthew R. James (S'86-M'88) was born in Sydney, Australia, on February 27, 1960. He received the B.Sc. degree in mathematics and the B.E. (Hon. I) in electrical engineering from the University of New South Wales, Sydney, Australia, in 1981 and 1983, respectively. He received the Ph.D degree in applied mathematics from the University of Maryland, College Park, in 1988.

From 1978 to 1984 he was employed by the Electricity Commission of New South Wales (now Pacific Power), Sydney, Australia. From 1985 to 1988 he held a fellowship with the Systems Research Center (now Institute for Systems Research), University of Maryland, College Park. In 1988 and 1989 he was Visiting Assistant Professor with the Division of Applied Mathematics, Brown University, Providence, RI, and from 1989 to 1991 he was Assistant Professor with the Department of Mathematics, University of Kentucky, Lexington. Since 1991 he has been Research Fellow with the Department of Systems Engineering, Australian National University, Canberra, Australia. Dr. James' research interests include nonlinear and stochastic systems, robotics, and advanced engineering computation. 


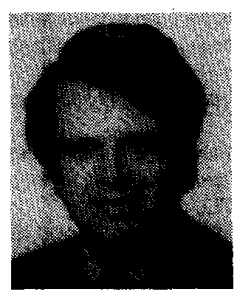

John S. Baras (S'73-M'73-SM'83'-F'84) was born in Piraeus, Greece, on March 13, 1948. He received the B.S. degree with highest distinction in electrical engineering from the National Technical University of Athens, Greece, in 1970 . He received the M.S. and Ph.D. degrees in Applied Mathematics from Harvard University, Cambridge, MA, in 1971 and 1973 respectively.

Since 1973, he has been with the Department of Electrical Engineering, University of Maryland at College Park, where he is currently Professor and member of the Applied Mathematics Faculty. From 1985 to 1991 he was the Founding Director of the Systems Research Center, now Institute for Systems Research. On February 1990 he was appointed to the Martin Marietta Chair in Systems Engineering. Since 1991 he has been the Codirector of the Center for Satellite and Hybrid Communication Networks, a NASA Center for the Commercial Development of Space, which he co-founded. He has held visiting research scholar positions with Stanford, MIT, Harvard University, the Institute National de Reserche en Informatique et en Automatique, and the University of California, Berkeley.

Dr. Baras has written numerous publications in control and communication systems and is the co-editor of Recent Progress in Stochastic Calculus (Springer-Verlag, 1990). Among his awards are: a 1978 Naval Research Laboratory Research Publication Award, 1980 Outstanding Paper Award of the IEEE Control Systems Society, and 1983 Alan Berman Research Publication Award from the Naval Research Laboratory. He has served in the IEEE Engineering R\&D Committee, the Aerospace Industries Association advisory committee on advanced sensors, the IEEE Fellow evaluation committee, and the IEEE Control Systems Society Board of Governors (1991-1993). $\mathrm{He}$ is currently serving on the editorial boards of Mathematics of Control Signals, and Systems, Systems and Control: Foundations and Applications, IMA Joumal of Mathematical Control and Information, Systems AutomationResearch and Applications and Information Systems from Springer-Verlag. $\mathrm{He}$ is a member of Sigma $\mathrm{Xi}$, the American Mathematical Society, and the Society for Industrial and Applied Mathematics.

His current research interests include stochastic systems, signal processing and understanding with emphasis on speech and image signals, real-time architectures, symbolic computation, intelligent control systems, robust nonlinear control, distributed parameter systems, hybrid communication network simulation and management.

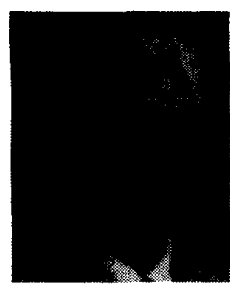

Robert Elliott received his Bachelors degree from Oxford, England in 1961, his Ph.D. from Cambridge, England in 1965 and an Sc.D. from Cambridge in 1983. He has been a Professor in the Department of Statistics and Applied Probability at the University of Alberta, Edmonton since 1986. Between 1977 and 1985 he held visiting positions at the University of Alberta, University of Kentucky and Brown University. He held the G.F. Grant Chair of Mathematics at the University of Hull in England from 1973 to 1986, and he was Head of Department from 1976. Prior to that period Dr. Elliott held positions at Yale, Oxford, Warwick and Northwestern.

Dr. Elliott's work is in signal and image processing and also in applications of random processes in finance. 